\title{
PDGFR-alpha inhibits melanoma growth via CXCL10/IP-10: a multi-omics approach
}

\author{
Daniela D'Arcangelo${ }^{1}$, Francesco Facchiano ${ }^{2}$, Giovanni Nassa ${ }^{3,4}$, Andrea Stancato ${ }^{1}$, \\ Annalisa Antonini ${ }^{1}$, Stefania Rossi ${ }^{2}$, Cinzia Senatore ${ }^{2}$, Martina Cordella ${ }^{2}$, Claudio \\ Tabolacci ${ }^{2}$, Annamaria Salvati ${ }^{3}$, Roberta Tarallo ${ }^{3}$, Alessandro Weisz ${ }^{3}$, Angelo M. \\ Facchiano $^{5}$, Antonio Facchiano ${ }^{1}$ \\ ${ }^{1}$ Istituto Dermopatico dell'Immacolata, IDI-IRCCS, Fondazione Luigi Maria Monti, Rome, Italy \\ ${ }^{2}$ Dipartimento Ematologia, Oncologia e Medicina Molecolare, Istituto Superiore di Sanità, Rome, Italy \\ ${ }^{3}$ Laboratory of Molecular Medicine and Genomics, Department of Medicine and Surgery, University of Salerno, Baronissi (SA), \\ Italy \\ ${ }^{4}$ Genomix4Life srl, Department of Medicine and Surgery, University of Salerno, Baronissi (SA), Italy \\ ${ }^{5}$ National Research Council Institute of Food Science, Avellino, Italy \\ Correspondence to: Daniela D'Arcangelo, email: d.darcangelo@idi.it \\ Antonio Facchiano, email: a.facchiano@idi.it \\ Keywords: cancer, omics, angiogenesis, miRNA, melanoma
}

Received: April 15, $2016 \quad$ Accepted: September 25, $2016 \quad$ Published: October 13, 2016

\section{ABSTRACT}

Melanoma is the most aggressive skin-cancer, showing high mortality at advanced stages. Platelet Derived Growth Factor Receptor-alpha (PDGFR-alpha) potently inhibits melanoma- and endothelium-proliferation and its expression is significantly reduced in melanoma-biopsies, suggesting that melanoma progression eliminates cells expressing PDGFR-alpha. In the present study transient overexpression of PDGFR-alpha in endothelial (HUVEC) and melanoma (SKMel-28, A375, Preyer) human-cells shows strong anti-proliferative effects, with profound transcriptome and miRNome deregulation. PDGFR-alpha overexpression strongly affects expression of 82 genes in HUVEC (41 up-, 41 down-regulated), and 52 genes in SKMel-28 (43 up-, 9 down-regulated). CXCL10/IP-10 transcript showed up to 20 fold-increase, with similar changes detectable at the protein level. miRNA expression profiling in cells overexpressing PDGFR-alpha identified 14 miRNAs up- and 40 down-regulated, with miR-503 being the most down-regulated ( 6.4 fold-reduction). miR-503, miR630 and miR-424 deregulation was confirmed by qRT-PCR. Interestingly, the most upregulated transcript (i.e., CXCL10/IP-10) was a validated miR-503 target and CXCL10/IP-10 neutralization significantly reverted the anti-proliferative action of PDGFR-alpha, and PDGFR-alpha inhibition by Dasatinb totally reverted the CXCL10/ IP10 induction, further supporting a functional interplay of these factors. Finally, integration of transcriptomics and miRNomics data highlighted several pathways affected by PDGFR-alpha.

This study demonstrates for the first time that PDGFR-alpha strongly inhibits endothelial and melanoma cells proliferation in a CXCL10/IP-10 dependent way, via miR-503 down-regulation.

\section{INTRODUCTION}

Cutaneous melanoma is the most aggressive skin cancer. Despite recent relevant therapeutic progresses, melanoma has still a poor prognosis at advanced clinical phases and contributes for the vast majority to skin cancers-related mortality. Multiple factors have been involved in the melanoma pathogenesis. A clear role has been identified for the BRAF mutational state along with sun exposure [1], intentional tanning [2] and exposure to other environmental pollutions such as insecticides and occupational exposures [3]. Increasing evidence 
is being collected indicating a role of the immunity, which appears to be impaired at least to some extent in melanoma [4]. To this regard, it should be highlighted that one of the recent advances in melanoma therapy refers to Ipilimumab [5], a CTLA-4 inhibitor acting as an immune response activator. Immune response is under the control of a complex molecular-network, including cytokines and chemokines [6], vitamins, interferon and interferon-induced proteins such as Interferon (IFN)- $\gamma$-induced protein 10 (CXCL10/ IP-10) [7, 8]. Inflammatory response is a key factor controlling initiation and progression of melanoma as well as other tumors [9, 10]; it is known to contribute to tumor development and anti-inflammatory agents are known to inhibit cancer growth, representing a class of novel potential anti-melanoma drugs [11]. Inflammation and immune response cooperate at different molecular levels to maintain the homeostasis and to control cancer development [12]. Several factors controlling inflammation and immune response have been identified to play regulatory actions in melanoma, such as Platelet Activating Factor (PAF) [9], microphthalmia-associated transcription factor (MITF) [13], granulocytemacrophage colony-stimulating factor [14], TNF-alpha [15], transforming growth factor [16]. CXCL10/IP-10 has a clear controlling role on immune response and inflammation [17-20] and has been recently found to be involved in liver and renal cancer development [21, 22]. It has been reported to inhibit melanoma growth and angiogenensis [23-26] and melanoma represses its expression in a nitric oxide-related manner [27].

Several growth factors, including Platelet Derved Growth Factors (PDGFs), have been shown to play a critical role in controlling angiogenesis as well as proliferation and metastatic potential of melanoma [2832]. The PDGF family exerts mitogenic and chemotactic actions in many cell types and with different potency. Such factors are present in 5 homo- and hetero-dimeric complexes (PDGF-AA, PDGF-BB, PDGF-AB, PDGF$\mathrm{CC}, \mathrm{PDGF}-\mathrm{DD})$ and bind to 3 dimeric receptors, namely PDGFRalpha/alpha (binding PDGFAA, PDGF-AB, PDGF-CC), PDGFRbeta/beta (binding PDGF-BB and PDGF-DD) and PDGF-Ralpha/beta (binding PDGF-AB, PDGF-BB, PDGF-CC, PDGF-DD) [33, 34]. As recently highlighted, the specific role such receptors play in cancer development and progression has still to be largely clarified [35]. The stimulatory role of PDGF$\mathrm{BB}$ and PDGFR-beta has been demonstrated in several cells and tissues, including melanoma [36-40]. Nonselective inhibitors of both PDGFR-beta and PDGFRalpha, such as imatinib and dasatinib, are known to partially inhibit melanoma growth, but the role of the alpha or beta receptors has not been fully clarified yet [41-43].

PDGF-AA and PDGFR-alpha are less potent mitogens, with contrasting- even inhibitory-effects in different cells and tissues, including endothelial and melanoma cells, as demonstrated in previous studies by ours and other laboratories [31, 44-46]. A recent study highlighted an inhibitory effect of PDGFR-alpha in ulcer healing, indicating a PDGFR-alpha mediated antiangiogenesis effect [47].

We have previously shown that PDGF-AA inhibits PDGF-BB angiogenic effects [28], and showed a direct interplay of Fibroblast Growth Factor-2 (FGF2) and PDGFs pathways, by demonstrating high-affinity binding of FGF-2 to PDGF-BB [48] [ and FGF-receptor/PDGFRalpha heterodimers formation [30]. We have also shown that PDGFR-alpha inhibits cell-growth both in vitro and in vivo, in endothelial- [29] as well as melanoma cells [31]. Such evidence shows a relevant anti-proliferation activity of PDGFR-alpha in melanoma; its expression has been found to be lost in human melanoma biopsies, likely due to a selection pressure acting to eliminate inhibitory factors. Further, over-expressing PDGFRalpha in melanoma reduces proliferation, inhibits DNA synthesis and increases apoptosis, cell cycle arrest and pRb dephosphorylation [31].

In the present study we better elucidate the molecular mechanisms underlying the PDGFR-alpha mediated antimelanoma effect. Further, transcriptomics and miRNomics data from human melanoma and endothelial cells overexpressing PDGFR-alpha were combined in a multiomics analysis, leading to the identification of pathways and functions involved in the anti-melanoma action of PDGFR-alpha overexpression.

\section{RESULTS}

\section{PDGFR-alpha overexpression inhibits endothelial and melanoma cells in vitro proliferation}

We previously demonstrated a strong antiproliferative and pro-apoptotic effect of PDGFR-alpha signaling, in human and mouse melanoma cells, and human endothelial cells (HUVEC) [30, 31]. In the present study we confirmed the strong anti-proliferative effect in human endothelial and in a larger panel of human melanoma cells, and then addressed in deeper details the mechanisms underlying such anti-proliferation effect. HUVEC and human melanoma (SKMel-28) cells were infected with increasing doses of PDGFR-alpha coding adenovirus (AdCMV.PDGFR-alpha) and control cells were infected with a null virus (AdCMV.null) (10, 30, $100 \mathrm{MOI})$. Figure $1 \mathrm{~A}$ and $1 \mathrm{~B}$ show that PDGFRalpha overexpression has a marked dose-dependent anti-proliferative effect, reaching $60 \%$ and $75 \%$ growth inhibition, in HUVEC and SKMel-28 cells, respectively. Figure 1C shows that such biological effect is confirmed in two additional aggressive human melanoma cells, namely A375 and Preyer cells. The overexpression of PDGFRalpha occurring upon $30 \mathrm{MOI}$ infection dose was then 
verified at the mRNA level by qRT-PCR (Supplementary Figure S1) and at the protein level (not shown).

\section{PDGFR-alpha expression in human normal skin biopsies and in melanoma biopsies}

Figure 1A-1C demonstrates that PDGFR-alpha overexpression inhibits proliferation in endothelial cells and in at least 3 human melanoma cells lines. We therefore hypothesized that PDGFR-alpha expression should be progressively lost in melanoma as compared to normal skin, as a mechanism to negatively select its inhibitory effect. Such hypothesis was tested by evaluating the expression levels of PDGFR-alpha in 355 human specimens, namely 208 skin melanoma and 147 normal skin, available at IST online (http:// ist.medisapiens.com). Figure 1D shows that PDGFR- alpha expression (reported onto the $\mathrm{X}$ axis) in melanoma biopsies (black circles) is strongly reduced as compared to normal skin samples (open circles). On the $\mathrm{Y}$ axis the expression of beta 2-microglobulin was used as reference. This is consistent with previous evidences showing a clear reduction of PDGFR-alpha expression in melanoma as compared to nevi sections [29]. In Figure $1 \mathrm{E}$ a further analysis carried out onto GEO database (http://www.ncbi.nlm.nih.gov/pubmed) indicates that melanoma progression from a primary stage toward a metastatic stage shows a further significant decrease of PDGFR-alpha expression. GDS3966 dataset [49] reports gene-expression profiling in 31 primary melanoma biopsies and in 52 metastatic melanoma biopsies. While the exact amount of neoplastic cells present in the different samples is not available for an accurate normalization, nevertheless mean PDGFR-
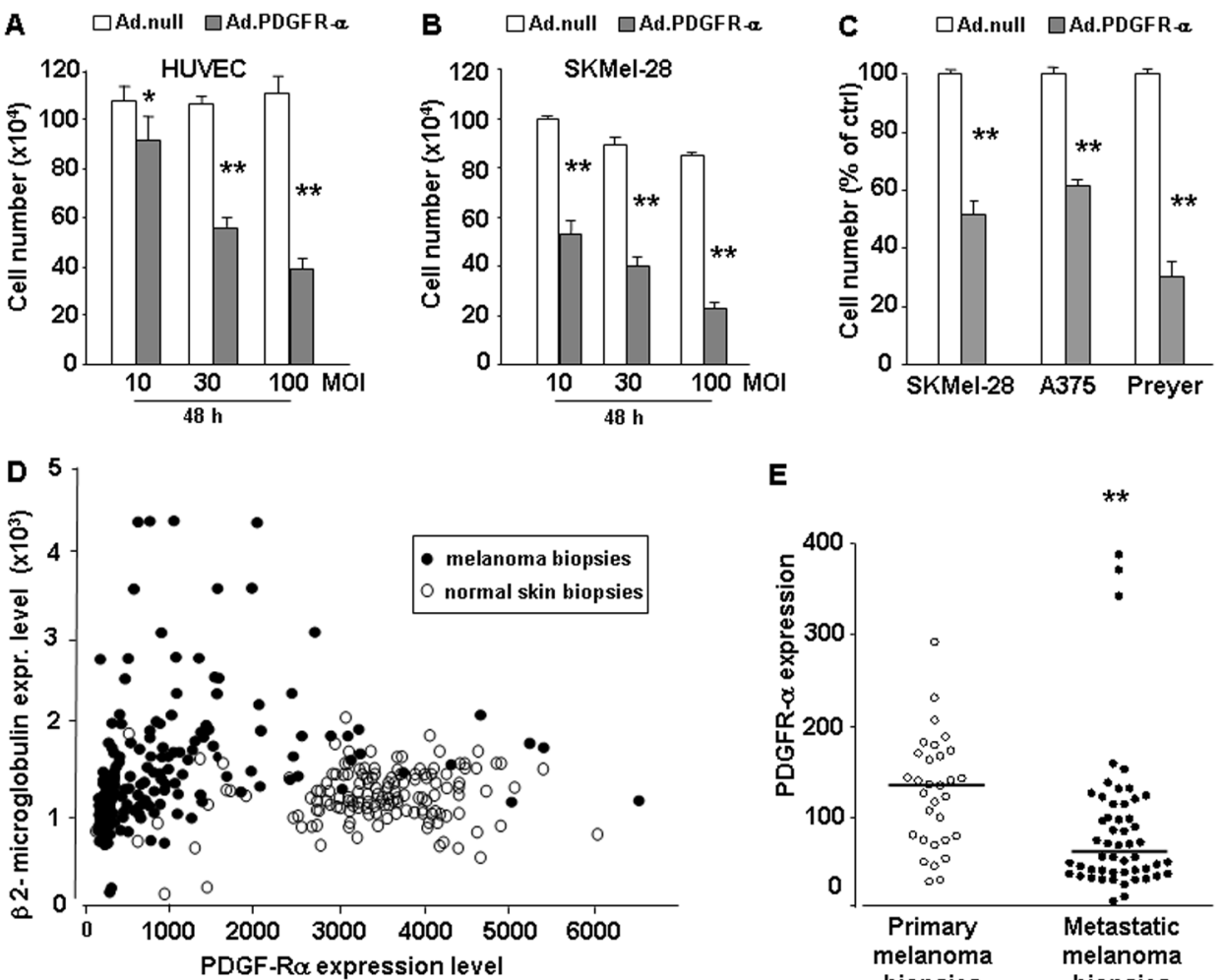

E

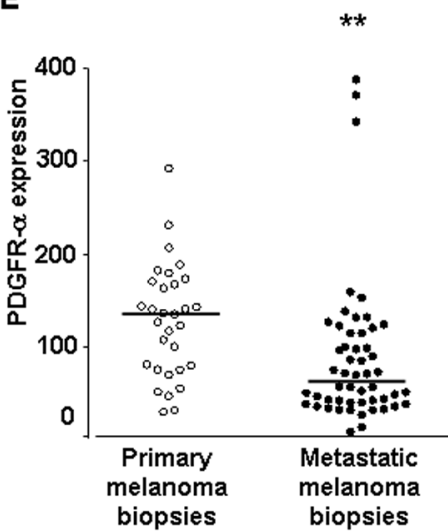

Figure 1: Effect of PDGFR-alpha overexpression on endothelial (HUVEC) and melanoma (SKMel-28) cells proliferation. Proliferation of HUVEC and SKMel-28 infected with Ad-vector codingfor PDGFR-alpha. A, B.: Dose-dependent effects of 10, 30, and 100 MOI AdCMV.PDGFR-alpha infection; 10\% FCS-induced proliferation at 48 hours. ${ }^{*} p$ value $<0.05$ and $* * p$ value $<0.01$ versus AdCMV.null (cell number at $\mathrm{T}=0$ corresponds to $7.5 \times 10^{5}$ ). Data are reported as mean $\pm \mathrm{SD}$ of 3 independent experiments. C. Effects of AdCMV.PDGFR-alpha infection in SKMel-28, A375 and Preyer cell lines. Data are reported as \% of control D. Expression of PDGFR-alpha (X axis) vs expression of the housekeeping gene beta 2-microglobulin (Y axis) in 208 melanoma biopsies (black spots) and 147 normal skin biopsies. Expression data were derived from ist.medisapiens.com site. Supplementary Figure 2SA-2SB reports correlation plots of PDGFR-alpha with other housekeeping genes, namely Tubulin Beta 1 gene (Supplementary Figure 2SA) and Actin beta gene (Fig. 2SB). In all cases PDGFR-alpha is strongly reduced in melanoma samples vs normal skin samples. E. Mean PDGFR-alpha expression in 31 primary human melanoma biopsies and in 52 metastatic human melanoma biopsies is significantly reduced $(\mathrm{p}=0.0002)$. Analysis carried onto GEO database, GDS3966 dataset (Xu 2008) (http://www.ncbi.nlm.nih.gov/pubmed). Expression values are reported. Graphing the "ranks values" of PDGFR-alpha (instead of the "uncorrected values") does not modify the significant reduction in metastatic vs primary samples $(0.58$ vs $0.73, p=0.0004)$. Beta 2-microglobulin, beta actin or tubulin beta1 ranks are very stable, invariantly 99 or 100 in all melanoma samples, independently from the "primary" or "metastatic" diagnosis. 
alpha expression was found to be significantly reduced $(\mathrm{p}=0.0002)$. Immunohistochemistry data available at Human Protein Atlas (http://www.proteinatlas.org/) confirm that PDGFR-alpha expression is undetected in most cancers (http://www.proteinatlas.org/ ENSG00000134853-PDGFRA/cancer) while PDGFRbeta is much more expressed (http://www.proteinatlas. org/ENSG00000113721-PDGFRB/cancer). More specifically to melanoma, PDGFR-alpha is undetected in $66 \%$ of melanoma tissue sections, while PDGFR-beta is undetected in $43 \%$ of melanoma tissue sections.

Data of Figure 1D and 1E support the hypothesis that melanoma onset and progression in vivo selects cells expressing lower levels of PDGFR-alpha.

\section{Gene expression profiling and validation in endothelial and melanoma cells over-expressing PDGFR-alpha}

In order to characterize the molecular mechanisms underlying the observed biological effect, gene expression profiling was evaluated in both HUVEC and melanoma cells over-expressing PDGFR-alpha, by Illumina microarray technology. HUVEC and SKMel-28 cells were infected with 30 MOI AdCMV.PDGFR-alpha; the same dose of the AdCMV.null virus was used to infect control cells. A value of at least 1.5 fold increase or decrease combined with a $\mathrm{p} \leq 0.001$ was chosen as relevant and significant threshold. In HUVEC overexpressing PDGFRalpha, the top up- and top down-regulated transcripts (41 in each case) were identified (Figure 2A; the complete list of 216 differentially modulated transcripts is reported in Supplementary Table S1). On the other hand, the top up- and top down-regulated transcripts (43 and 9, respectively) in the SKMel-28 melanoma cells overexpressing PDGFR-alpha are shown in Figure 2B (the complete list of 107 differentially modulated transcripts is reported in Supplementary Table S2). Figure 2A and 2B show that, upon PDGFR-alpha overexpression, a number of genes undergo a similar regulation in HUVEC and in melanoma cells. Namely, CXCL10/IP-10 gene is the most up-regulated in SKMel-28 and the second most up-
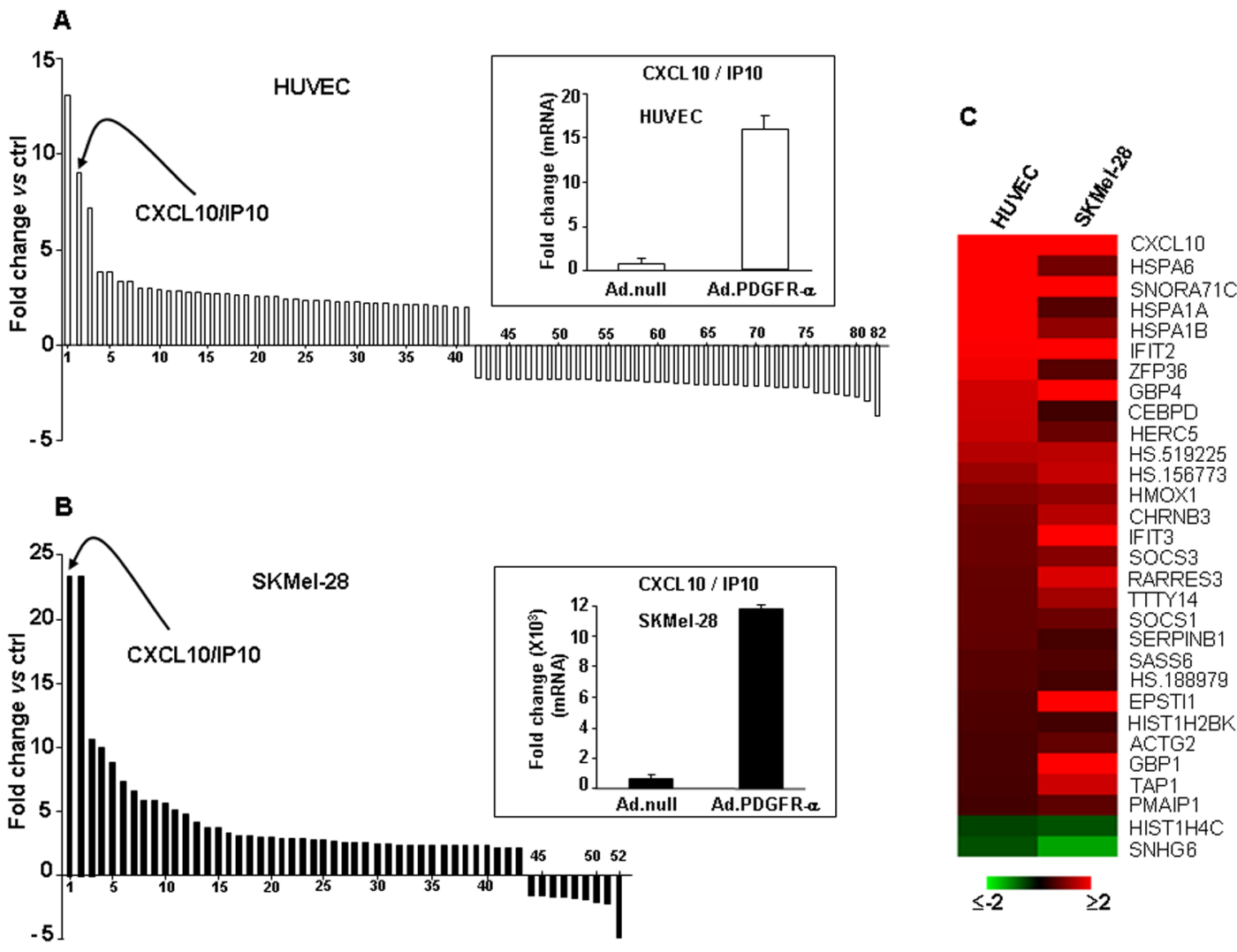

Figure 2: Gene expression profiling and validation in endothelial cells and melanoma overexperessing PDGFR-alpha. A. Bar graph depicts the top 41 transcripts up-regulated and top 41 down-regulated in HUVEC. Inset reports the corresponding validation by qRT-PCR. Data are reported as mean \pm SD of 3 independent experiments. The complete list of 216 differentially modulated transcripts is reported in Supplementary Table S1. B. Bar graph depicts the top 43 transcripts up-regulated and top 9 down-regulated in SKMel-28. CXCL10/IP-10 transcript was found strongly up-regulated both in HUVEC and SKMel-28. Inset reports the corresponding validation by qRT-PCR, carried out as 3 independent experiments. Data are reported as mean \pm SD. The complete list of 107 differentially modulated transcripts is reported in Supplementary Table S2). C. Heatmap depicting all common differentially regulated transcripts in HUVEC and SKMel-28 over-expressing PDGFR-alpha as compared to Ad.null infected cells. 
regulated in HUVEC (23 and 9 fold-increase, indicated by the arrows, respectively). Further, SNORA71C, IFT2 and GBP4 genes are among the most up-regulated in both cells lines (see Supplementary Table S1 and S2). All common differentially expressed transcripts are reported in Figure 2C. Modulation of CXCL10/IP-10 geneexpression reported in Figure 2 was then validated by mRNA quantification in qRT-PCR on an independent set of experiments. Insets of Figure $2 \mathrm{~A}$ and $2 \mathrm{~B}$ confirm that PDGFR-alpha overexpression induces a highly significant increase of CXCL10/IP-10 mRNA expressions in both HUVEC (Figure 2A inset) and SKMel-28 melanoma cells (Figure 2B inset). Expression of the unique receptor of CXCL10/IP10 (namely, CXCR3) was found to be not modulated in HUVEC nor in SKMel-28 overexpressing PDGFR-alpha, in both gene expression profiling and qRTPCR (not shown).

\section{CXCL10/IP-10 protein expression in endothelial and melanoma cells overexpressing PDGFR- alpha}

Since CXCL10/IP-10 was found strongly upregulated at the mRNA level in both HUVEC and melanoma cells (Figure 2A and 2B), we aimed at validating such modulation at the protein level. Cytokine expression was thus measured in cell lysates obtained from HUVEC and SKMel-28 cells overexpressing PDGFR-alpha. Figure 3A and 3B indicate that CXCL10/ IP-10 protein is strongly and significantly up-regulated in PDGFR-alpha-overexpressing cells, as compared to control. A similarly strong and significant effect was then confirmed in two additional human melanoma cells overexpressing PDGFR-alpha, namely A375 and Preyer (Figure 3C and 3D). Such results indicate that transient overexpression of PDGFR-alpha is associated to CXCL10/ IP-10 increased expression by several fold vs control, namely, 20 fold in HUVEC, 41 fold in SKMel-28, 103 fold in A375 and 7.6 fold in Preyer cells.

\section{miRNA expression in melanoma and endothelial cells overexpressing PDGFR-alpha. Profiling experiments and $q R T-P C R$ validation}

In addition to gene-expression profiling and protein-level analysis, miRNAs profile in PDGFR-alpha overexpressing cells (HUVEC and SKMel-28) was then analyzed by a miRNA microarray Agilent platform, measuring the expression of over 900 miRNAs. Figure 4A shows that overexpressing PDGFR-alpha leads to the significant up-regulation of 14 miRNAs in HUVEC. The most up-regulated is miR-630 (more than 12 fold, $\mathrm{p}=0.0006$ vs control). On the other hand, 39 miRNAs were found to be significantly reduced; the most downregulated is miR-503 (6.4 fold reduction, $p=0.016$ vs control). Interestingly enough, miR-424, another member of the miR-503 cluster, was also found down-regulated about 2 fold. In SKMel-28 cells, the overexpression of PDGFR-alpha leads to the significant up-regulation of 11 miRNAs and reduction of 10 miRNAs (Figure 4C). Nine miRNAs resulted to be commonly differentially expressed between HUVEC and SKMel-28 cells
A

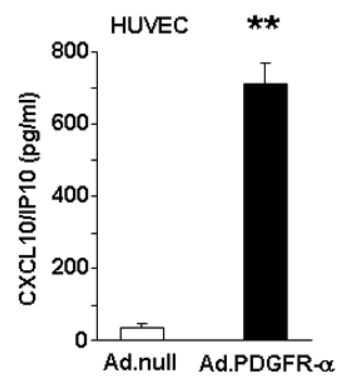

C

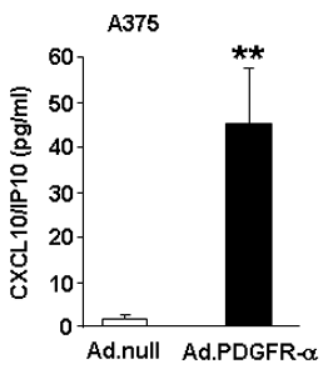

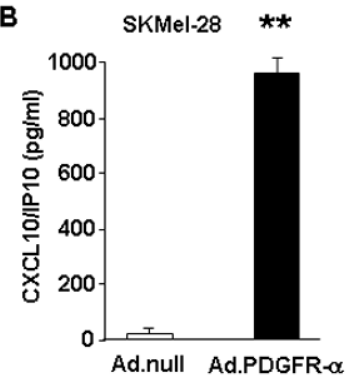

D

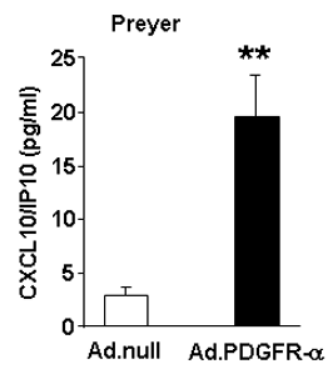

Figure 3: CXCL10/IP10 protein expression. CXCL10/IP-10 expression level measured in cell lysates obtained from endothelial (HUVEC) and melanoma (SKMel-28, A375, Preyer) cells over-expressing PDGFR-alpha, as compared to Ad.null-infected cells. Data are reported as mean $\pm \mathrm{SD}$ of 3 independent experiments and normalized by total protein content. 
as reported in the heatmap (Figure 4D). Validation is reported in Figure 4C. qRT-PCR analysis on an independent set of HUVEC and SKMEL-28 cells overexpressing PDGFR-alpha was carried out. miR-503 was confirmed to be strongly and significantly downregulated both in HUVEC and in SKMel-28 (fold-change -2 with $p=0.0008$ and $=0.004$, respectively). The upregulation of miR-630 and the down-regulation of miR424 reported in Figure 4A were also confirmed by qRTPCR in both HUVEC and SKMel-28 (Figure 4B). These data indicate that miR-503 is strongly down-regulated in both HUVEC and SKMEL-28 cells overexpressing PDGFR-alpha, as compared to Ad.CMV.null control cells. All differentially expressed miRNAs are reported in Supplementary Table S3 and Supplementary Table S4, along with the exact fold changes and computed $p$ values.

\section{mir-503 target prediction and target validation}

Figure 2 and Figure 3 show the relevant increased expression of CXCL10/IP-10 at both mRNA and protein levels, as result of PDGFR-alpha overexpression. Figure 4A and 4B show the strong reduction miR-503 expression in the same experimental set up. Therefore we hypothesized a functional interplay between CXCL10/
IP-10 and miR-503, downstream the PDGFR-alpha. According to TargetScan software, CXCL10/IP-10 is one of the predicted miR-503 targets, given the computed high score of complementarity of CXCL10/ IP-10 3'UTR and miR-503 seed sequence (Figure 5A). To experimentally confirm such prediction a 3 '-UTRLuc assay was carried out. A transient transfection of 3'-UTR CXCL10/IP-10 Luciferase stable 293 Cell Line with miR-503 led to a significant decrease (about $50 \%, \mathrm{p}<0.0001)$ in Luciferase reporter expression as compared to the control vector (Figure 5B). Such effect was significantly reverted when a deleted form of the 3'-UTR CXCL10/IP-10 was used as specificity control (Figure 5B), demonstrating that the 3 '-UTR CXCL10/ IP-10 is a functional target of miR-503, as predicted by TargetScan.

\section{CXCL10/IP-10 neutralization reverts PDGFR- alpha-dependent growth inhibition and dasatinb reverts PDGFR-alpha dependent overexpression of CXCL10/IP10}

Figures 2 to 5 represent strong evidences allowing us to hypothesize that CXCL10/IP-10 and miR-503 are involved in the PDGFR-alpha anti-proliferation activity
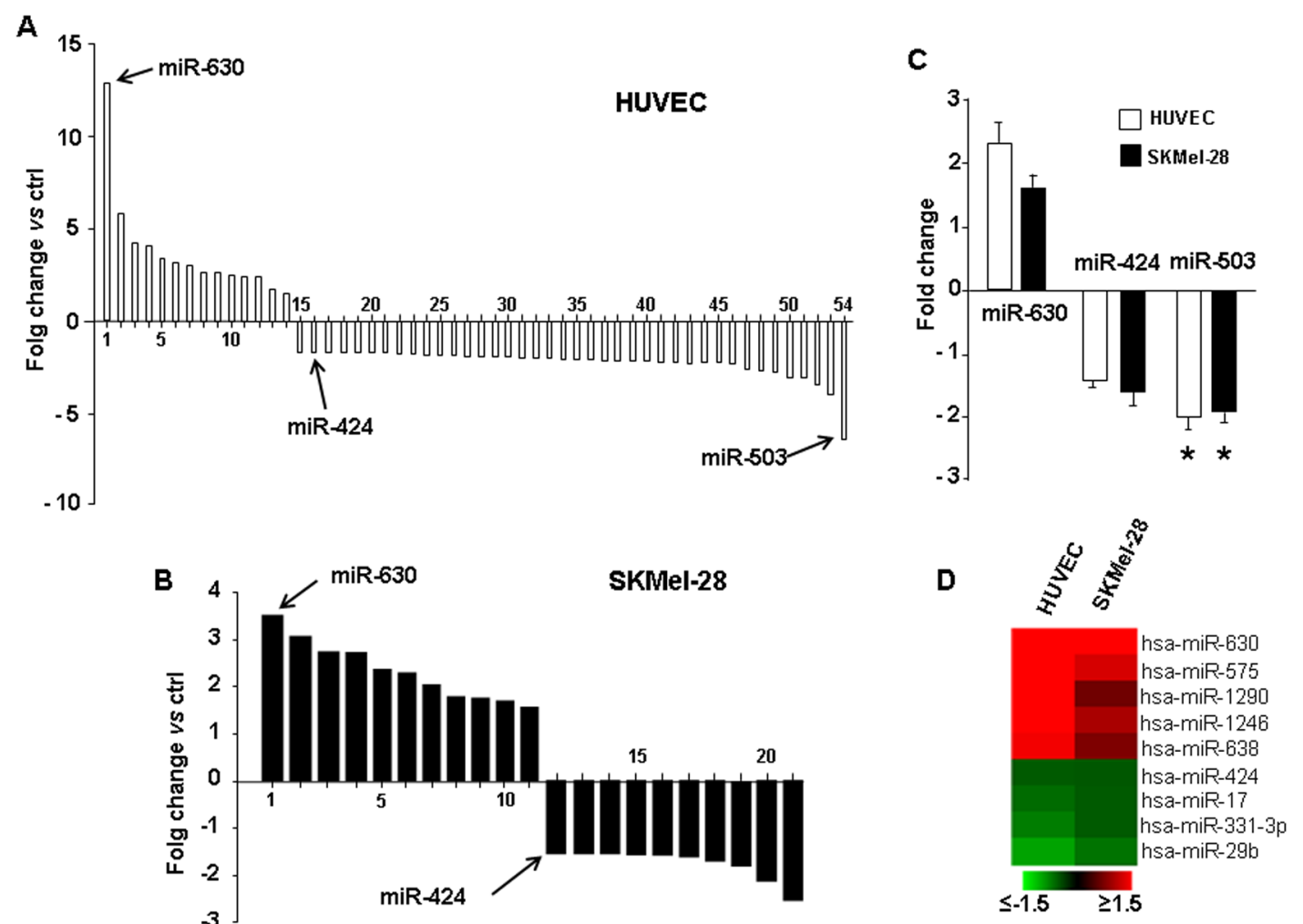

Figure 4: miRNA profiling and qRT-PCR validation. miRNome profiling in HUVEC A. and SKMel-28 cells B. overexpressin PDGFR-alpha vs Ad.null control cells. The complete list of all differentially expressed miRNAs is reported in Supplementary Table S3 and Supplementary Table S4, along with the exact fold changes and computed p values. C. qRT-PCR validation, in HUVEC and SKMel-28 cells overexpressing PDGFR-alpha. Data are reported as mean \pm SD of 3 independent experiments. D. Heatmap depicting all common differentially regulated miRNAs in HUVEC and SKMel-28 over-expressing PDGFR-alpha as compared to Ad.null infected cells. 
A
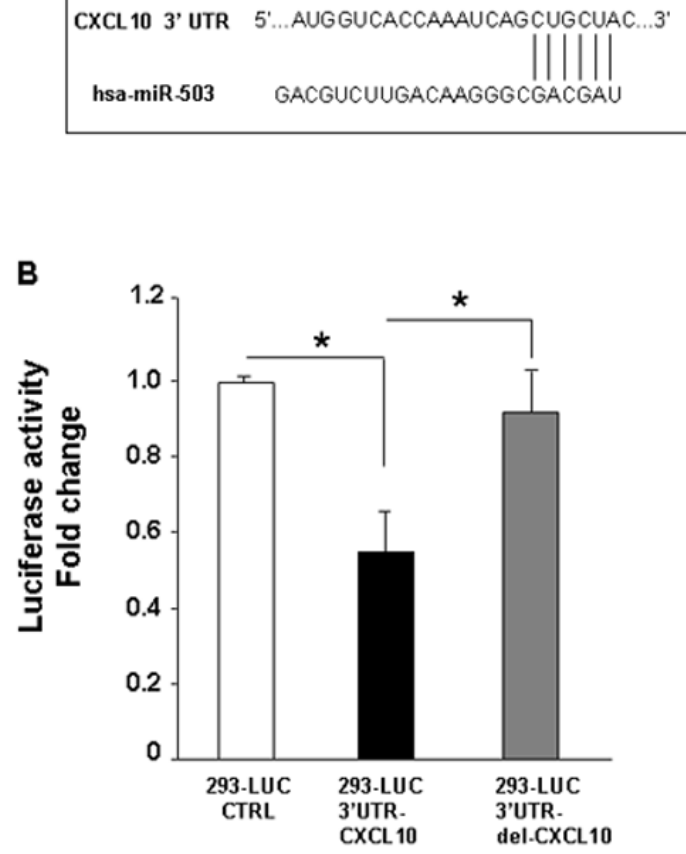

Figure 5: Target prediction of miR-503 and validation. A. CXCL10/IP-10 was predicted to be one of the miR-503 targets according to TargetScan software, given the complementarity of CXCL10/IP-10 3'UTR and miR-503 seed sequence. B. 3'-UTR-Luc assay shows a significant decrease (about 50\%, $p$ value $<0.0001$ ) in Luciferase reporter expression in transiently transfected 3'-UTR CXCL10/ IP10 Luciferase stable 293 cell line with miR-503, as compared to the control vector, and a significant reversion is observed when a deleted form of the 3'-UTR CXCL10/IP-10 was used as specificity control. Data are reported as mean \pm SD of 3 independent experiments.
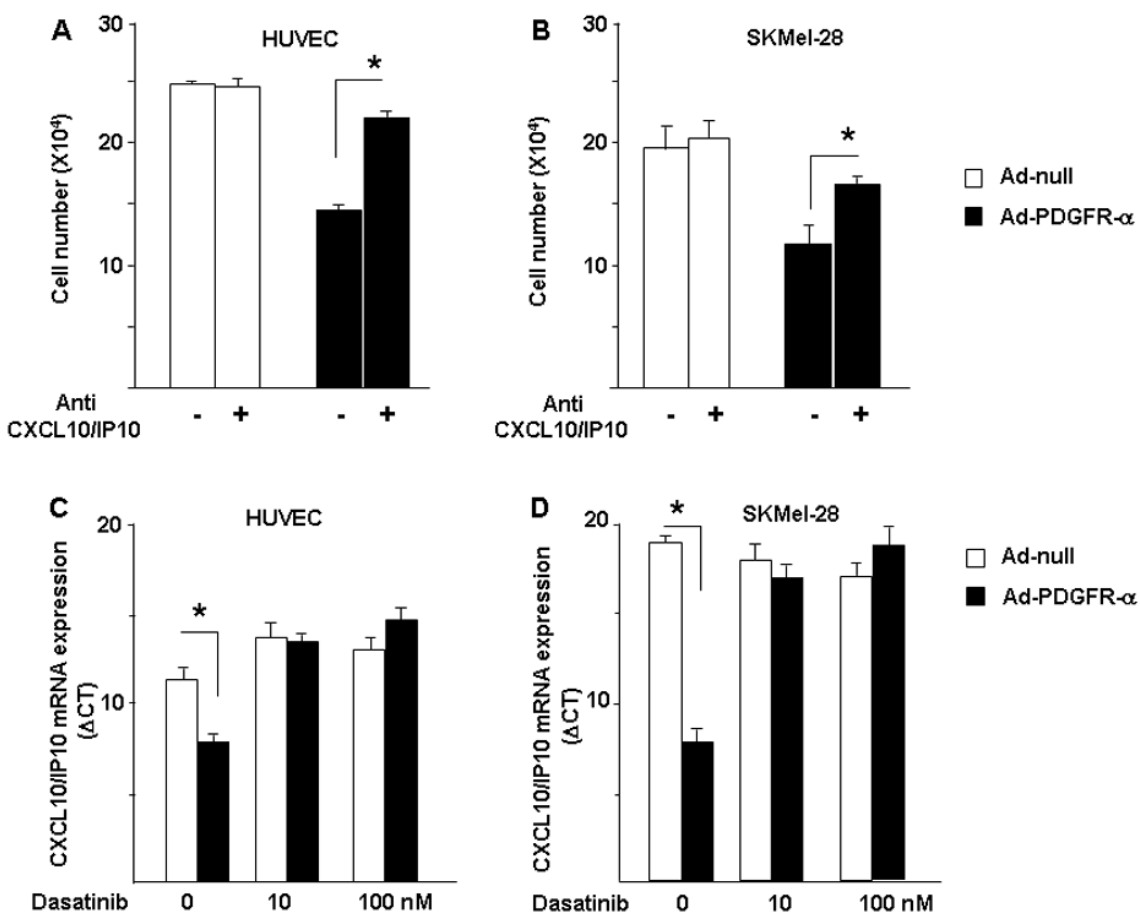

Figure 6: Mechanisms characterization. Neutralization of CXCL10/IP10 reverts PDGFR-alpha-dependent growth inhibition. The key role of CXCL10/IP-10 to mediate the anti-proliferation effect of PDGFR-alpha was confirmed by the use of the neutralizing antibody anti-CXCL10/IP-10, which partially but significantly reverts the inhibitory effect of PDGR-alpha overexpression, in both HUVEC A. and SKMel-28 B. cells. The anti-tumor drug Dasatinb, known inhibitor of PDGFR-alpha and other tyrosine-kinases, completely restores CXCL10/IP10 expression (expressed as $\triangle \mathrm{CT}$ ). Data are reported as mean $\pm \mathrm{SD}$ of 3 independent experiments. 
shown in Figure 1. Cells overexpressing PDGFRalpha were then exposed to the specific neutralization of CXCL10/IP-10. As reported in Figure 6A and 6B, treatment with the neutralizing CXCL10/IP-10 antibody significantly reverted the growth inhibition given by PDFR-alpha overexpression, in both HUVEC and SKMel-28 cells. Such results allowed us to conclude that CXCL10/IP-10 represents a key player of the anti-proliferation action of PDGFR-alpha. To further characterize the observed effects, we used an antitumor drug currently in clinical practice, namely Dasatinib. It is a known inhibitor of PDGFR-alpha and other tyrosin kinase receptors. Under Dasatinb treatment, the CXC110/IP10 upregulation induced by PDGFR-alpha was completely reverted, further suggesting that the observed modulation of CXCL10/IP10 is PDGFR-alpha related.

\section{Integrated omics analysis}

An exhaustive bio-informatic analysis was then carried out to integrate the obtained transcriptomics and miRNomics data. According to the stringent criteria reported in Materials and Methods section, genes and miRNAs up- and down-regulated vs the corresponding controls, in cells over-expressing PDGFR-alpha, were analyzed to predict the affected molecular pathways. Such analysis was carried out with two different bio-informatics approaches: i.e, via KEGG pathways analysis and via Ingenuity Pathway Analysis (IPA) software. KEGG analysis showed that HUVEC over-expressing PDGFR-alpha undergo the significant up-regulation of 13 molecular pathways, and the significant down-regulation of 5 molecular pathways, listed in Table 1. On the other hand, KEGG analysis revealed that SKMel-28 cells over-expressing PDGFR-alpha undergo the significant up-regulation of 5 molecular pathways (4 of which being upregulated also in HUVEC), while no pathways were found to be significantly down-regulated. The Cytokine-Cytokine receptor interaction pathway was the most strongly affected both in HUVEC and in SKMel-28 cells. In fact, the expression of 9 genes and 6 genes of this pathway was modified in HUVEC and SKMel-28 in a significant manner $(\mathrm{p}=1 \times 10-11$ and $\mathrm{p}=6 \times 10-7$ respectively) (see Table 1).

An additional approach was carried out with the IPA analysis, which highlighted the relevant effect of PDGFR-alpha overexpression on several molecular functions, including Cell Signaling, Cell Growth and Proliferation, Cell Development, Cell Movement, Cell Death and Survival, confirming the KEGG analysis results. The molecular functions significantly affected in HUVEC and SKMel-28 cells are reported in Word Cloud (Figure 7A and 7B, respectively). Supplementary Table S5A and S5B report the differentially expressed transcripts and the predicted affected Top Diseases and Functions by network they form, in HUVEC and SKMel-28, respectively, according to IPA functional annotation. Several Top Diseases and Functions are commonly affected in the two cell systems analyzed, such as Cancer, Inflammatory response, Hematological System Development and Function, Cell to Cell Signaling and Interaction, Tissue morphology, Cell cycle, Cell Death and Survival, Cardiovascular Disease. The Top Diseases and Functions identified with the highest score comprise Cancer, Cell Death and Survival and Inflammatory Response.

Data from Figure 2A and Figure 4 were further integrated. Namely, within the list of deregulated transcripts reported in Figure 2A, IPA software identified the highly predicted or experimentally validated targets of the miRNAs differentially expressed reported in Figure 4. Supplementary Table S6 reports the whole list of differentially expressed mRNAs targeted by differentially expressed miRNAs, in addition to CXCL10/IP-10. The miR-503/CXCL10/IP-10 represents the best match showing the highest miRNA down-regulation (namely, -6.39 fold decrease) and the highest mRNA transcript upregulation ( +9 fold increase).

The molecular pathways putatively affected by the miRNAs deregulation were then also predicted in PDGFR-alpha overexpressing HUVEC. As an example, Figure 8 shows that several transcripts found to be up- or down-regulated fall within the "Cell death and Survival" functional category. This Figure represents a narrow snapshot of the complex molecular status observed in cells overexpressing PDGFR-alpha. It shows the reduced levels of miR-503 and miR-424, the increased level of CXCL10/ IP-10, and the observed up- and down- regulation of other factors. The arrows report the known functional connections, according to IPA software database. Most interestingly, CEBPB and CEBPD transcriptor factors are found up-regulated. CEBPB shares functional interactions with CXCL10/IP-10 [50], and both CEBPB and CEBPD are known to be tumor suppressors [51-54]. Therefore their up-regulation in HUVEC overexpressing PDFRalpha may explain, at least in part, the observed antiproliferation effect.

\section{DISCUSSION}

We have previously shown a direct molecular and functional interplay between FGF-2 and PDGF family members, by demonstrating that such ligands interact with high affinity, inducing hetero-dimerization of the corresponding receptors [30], leading to a relevant inhibition of cell growth in both endothelial [55] and melanoma cells, either in vitro and in vivo mouse melanoma models [56]. Such inhibitory role of PDGFRalpha appears not totally surprising, given that the other main receptor of this family, PDGFR-beta, is highly 
Table 1: KEGG signaling pathways significantly affected by PDGFR-alpha over-expression, in HUVEC and in SKMel-28

\begin{tabular}{|c|c|c|c|c|c|}
\hline \multicolumn{6}{|c|}{ KEGG pathways } \\
\hline \multicolumn{6}{|c|}{ UP REGULATED IN HUVEC } \\
\hline & & $\begin{array}{l}\text { N. of genes found } \\
\text { to be regulated }\end{array}$ & $\begin{array}{l}\% \text { of all genes present } \\
\text { in the pathway }\end{array}$ & $\begin{array}{l}\text { t test } \\
\text { p value }\end{array}$ & $\begin{array}{l}\text { Benjamini } \\
\text { p value }\end{array}$ \\
\hline $\begin{array}{l}\text { Cytokine-cytokine receptor } \\
\text { interaction }\end{array}$ & $*$ & 9 & 8.6 & $1 \mathrm{E}-11$ & $8 \mathrm{E}-10$ \\
\hline Pathways in cancer & & 7 & 6.7 & $6 \mathrm{E}-7$ & $2 \mathrm{E}-5$ \\
\hline NOD-like receptor & & 5 & 4.8 & 2 E-6 & $6 \mathrm{E}-5$ \\
\hline Jak-STAT signaling & * & 5 & 4.8 & 5 E-6 & $8 \mathrm{E}-5$ \\
\hline Chemokine signaling pathway & $*$ & 5 & 4.8 & $2 \mathrm{E}-5$ & 3 E-4 \\
\hline Toll-like receptor & & 4 & 3.8 & $1 \mathrm{E}-4$ & 0.01 \\
\hline MAPK signaling pathway & & 5 & 4.8 & $1 \mathrm{E}-4$ & 0.01 \\
\hline TGF-beta signaling pathway & & 3 & 2.9 & 0.002 & 0.01 \\
\hline Bladder cancer & & 3 & 2.9 & 0.002 & 0.01 \\
\hline $\begin{array}{l}\text { Cell adhesion molecules } \\
\text { (CAMs) }\end{array}$ & & 3 & 2.9 & 0.002 & 0.01 \\
\hline $\begin{array}{l}\text { Neuroactive ligand-receptor } \\
\text { interaction }\end{array}$ & & 3 & 2.9 & 0.002 & 0.01 \\
\hline $\begin{array}{l}\text { Antigen processing and } \\
\text { presentation }\end{array}$ & $*$ & 3 & 2.9 & 0.003 & 0.02 \\
\hline Cell cycle & & 3 & 2.9 & 0.007 & 0.04 \\
\hline \multicolumn{6}{|c|}{ DOWN REGULATED IN HUVEC } \\
\hline Oocyte meiosis & & 3 & 3.6 & 5 E-4 & 0.002 \\
\hline $\begin{array}{l}\text { Ubiquitin mediated } \\
\text { proteolysis }\end{array}$ & & 3 & 3.6 & 0.002 & 0.005 \\
\hline Endocytosis & & 3 & 3.6 & 0.003 & 0.004 \\
\hline Cell cycle & & 3 & 3.6 & 0.003 & 0.004 \\
\hline Axon guidance & & 3 & 3.6 & 0.006 & 0.007 \\
\hline \multicolumn{6}{|c|}{ UP REGULATED IN SKMel-28 } \\
\hline $\begin{array}{l}\text { Cytokine-cytokine receptor } \\
\text { interaction }\end{array}$ & $*$ & 6 & 7 & $6 \mathrm{E}-7$ & $2.00 \mathrm{E}-05$ \\
\hline Chemokine signaling pathway & $*$ & 5 & 5.8 & $8.00 \mathrm{E}-06$ & $1.00 \mathrm{E}-04$ \\
\hline $\begin{array}{l}\text { RIG-I-like receptor signaling } \\
\text { pathway }\end{array}$ & & 3 & 3.5 & 7.00E-03 & 0.09 \\
\hline Jak-STAT signaling & $*$ & 3 & 3.5 & 0.007 & 0.09 \\
\hline $\begin{array}{l}\text { Antigen processing and } \\
\text { presentation }\end{array}$ & $*$ & 3 & 3.5 & 0.01 & 0.09 \\
\hline
\end{tabular}

DOWN REGULATED IN SKMel-28

No pathway

* Pathways upregulated both in HUVEC and SKMel-28 
A

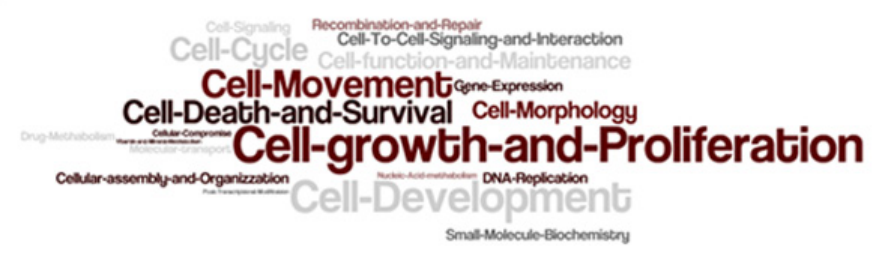

B

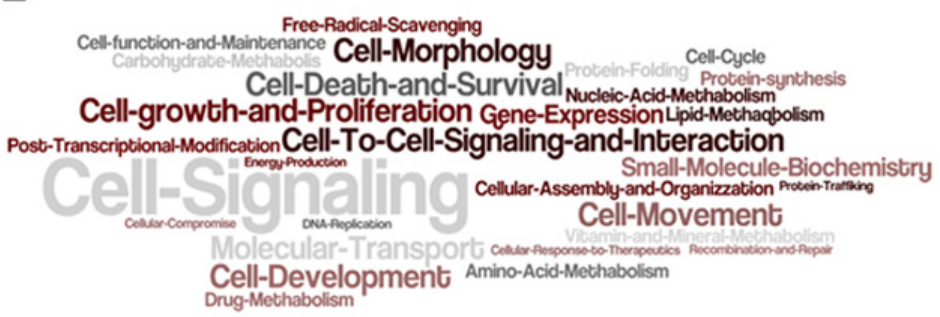

Figure 7: IPA Functional Analysis. Word Cloud analysis of significantly enriched molecular functions identified by IPA functional analysis considering differentially expressed transcripts in HUVEC A. and SKMel-28 cell B. over-expressing PDGFR-alpha. The text size is inversely proportional to the $\log p$ value $\leq 0.05$ (Fisher's exact test).

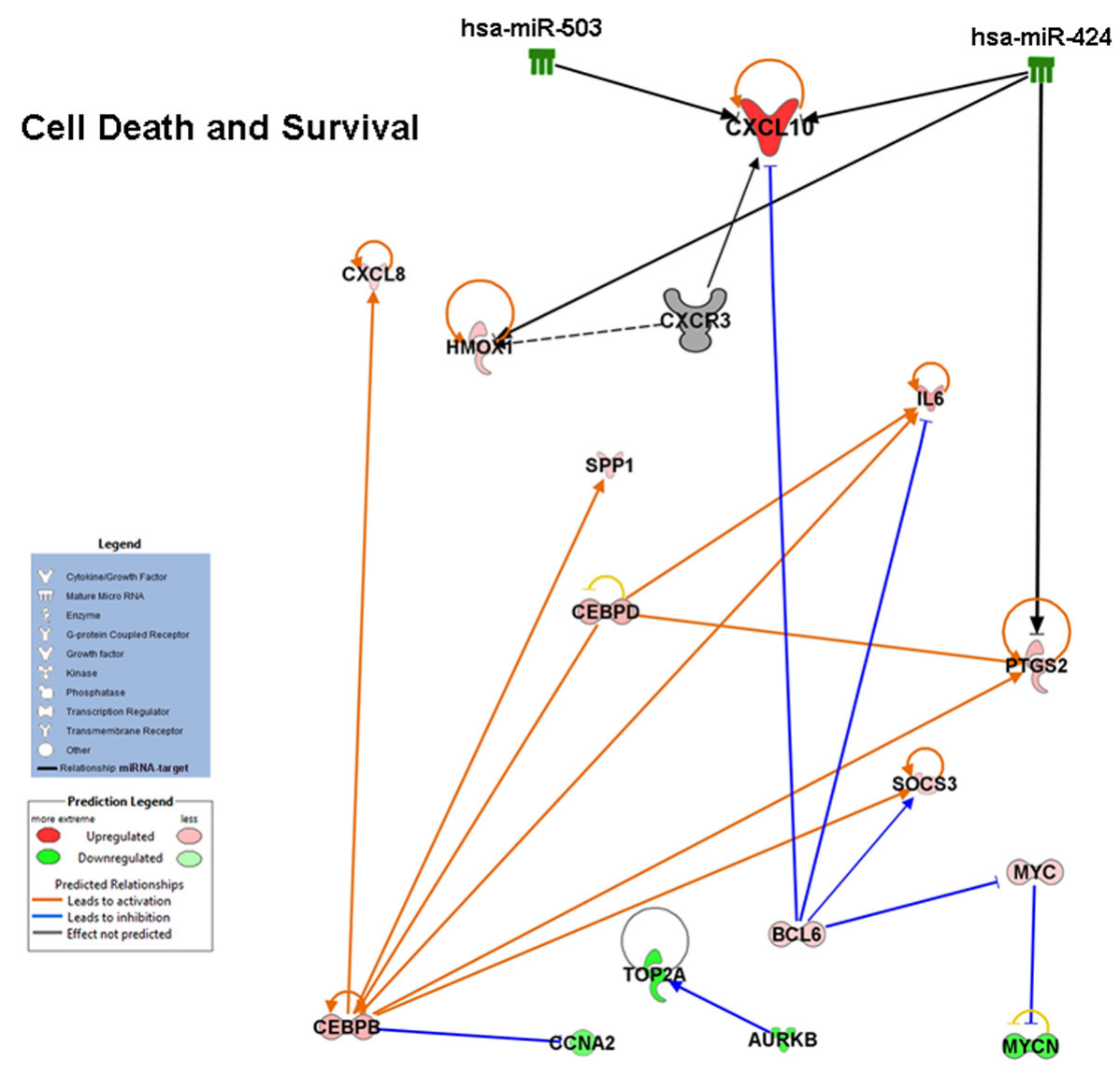

Figure 8: Integration of miRNA and transcriptome changes occurring in HUVEC cells. Functional network obtained by IPA analysis highlighting inverse relationships between miRNA and transcripts expression induced by PDGR-alpha overexpression. Gene expression- and miRNA- microarray data were integrated. 
Table 2: Primers used in the qRT-PCR analysis

human PDGFR-alpha Fw

human PDGFR-alpha Rev

human CXCL10/IP10 Fw

human CXCL10/IP10 Rev

human beta 2-microglobulin Fw

human beta 2-microglobulin Rev

human PDGFR-beta Fw

human PDGFR-beta Rev

human CXCR3 Fw

human CXCR-3 Rev

$$
\begin{gathered}
\text { 5'-tttgatttcttccagcattgtg-3', } \\
\text { 3'-aggtggttgaccttcaatgg-5', } \\
\text { 5'-gacatatactccatgtagggaagtga-3' } \\
\text { 3'-gaaagcagttagcaaggaaaggt-5' } \\
\text { 5'-ttctggcctggaggctatc-3' } \\
\text { 3'-tcaggaaatttgactttccattc-5' } \\
\text { 5'-gagacgttgatggatgacacc-3' } \\
\text { 5'-catctgcaaaaccaccattg-3' } \\
\text { 5'-caaccacaagcaccaaagc-3' } \\
\text { 5'-tcttctgcgtgatcccatc-3', }
\end{gathered}
$$

related to the v-SIS oncogene of simian sarcoma virus. While PDGFR-alpha has been recently investigated in melanoma surrounding fibroblast [44], in the current study we addressed PDGFR-alpha action in other key players within the melanoma tissue, i.e., endothelial and melanoma cells. Overexpressing PDGFR-alpha allowed us to force the signaling toward a PDGFR-alpha/alpha signaling and to follow biological and molecular effects mostly related to a pure PDGFR-alpha/alpha signal. Under such conditions, a clear anti-proliferation action in both endothelial and melanoma cells has been found, consistently with previous reports $[29,31,32]$.
The inhibitory role of PDGFR-alpha in melanoma was supported by data collected in several hundred melanoma patients' biopsies, showing a significant reduction of PDGFR-alpha expression in melanoma biopsies as compared to normal skin, as well as in metastatic melanoma biopsies as compared to primary melanoma biopsies (Figure 1D, 1E and datasets GDS1375, GDS1989, GDS2200, GDS1965 available at GEO database site http://www.ncbi.nlm.nih.gov/sites/entrez) [31]. This indicates that PDGFR-alpha is lost in melanoma tissue, likely due to its inhibitory effect. A negative selection process may therefore occur, thus supporting the

\section{Pathways affected (obsenved)}

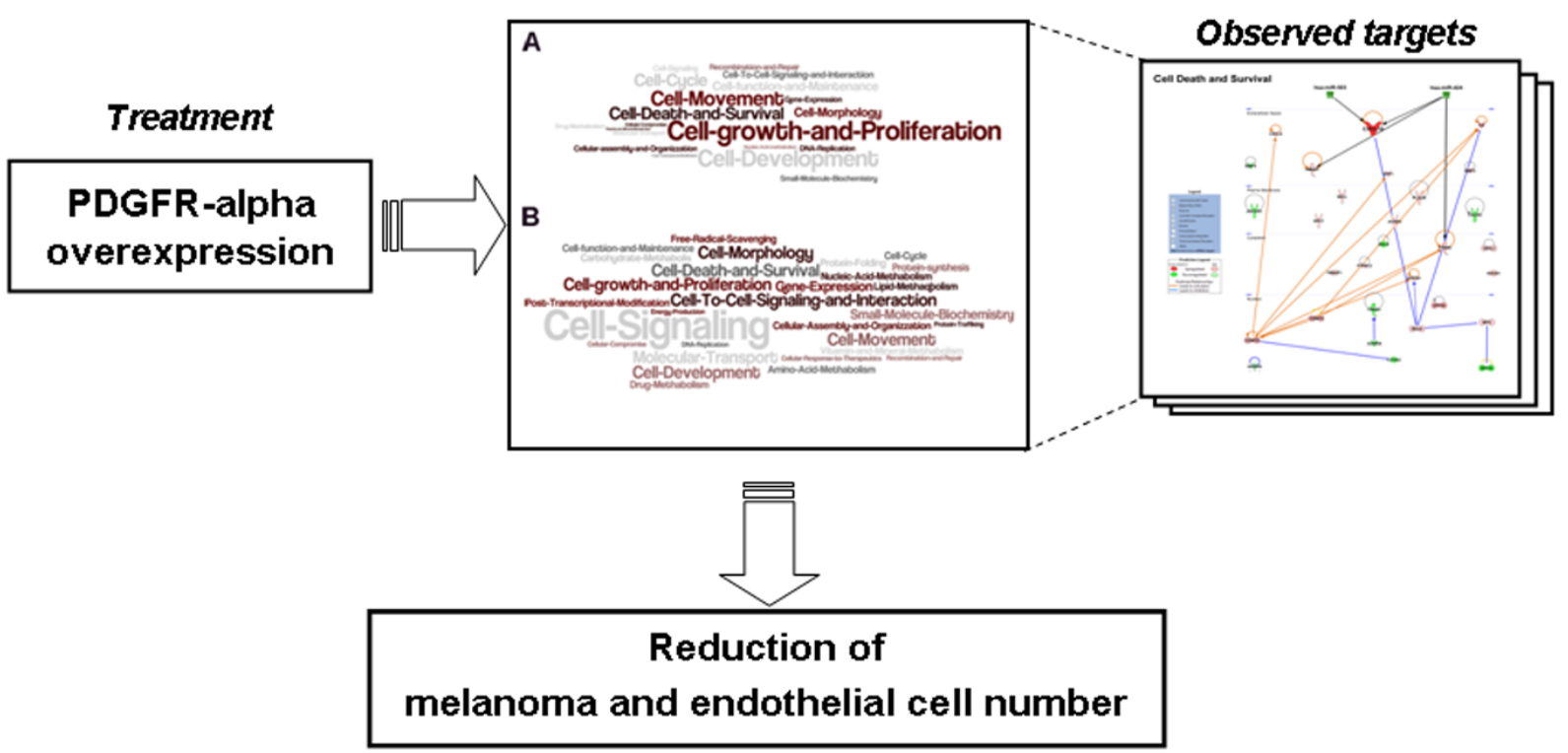

\section{Biological effects (observed)}

Figure 9: Cartoon summarizing the intervention and the cellular reactions observed in the current study. Overexpressing PDGFR-alpha induces reduction of melanoma and endothelial cell number affecting several molecular functions via up- and down- regulation of several interconnected molecules. 
hypothesis that melanoma progression in vivo may select cells expressing low or very-low levels of PDGFR-alpha.

The current study shows that overexpression of PDGFR-alpha is associated with a strong induction of the pro-inflammatory cytokine CXCL10/IP-10 (Figure 2 and Figure 3). Inflammation appears as one of the most affected function in cells over-expressing PDGFR-alpha (Supplementary Table S5A and S5B). This finding is consistent with previous data showing that the onset of an appropriate inflammatory response may finally exert anti-tumor activity [57]. Figure 8 shows the functional interconnections of different pro-inflammatory factors upregulated in PDGFR-alpha over-expressing cells, such as IL6 and CXCL8, further suggesting the hypothesis that an inflammatory response may be involved in the observed anti-angiogenesis and anti-melanoma effect of PDGFRalpha. Interestingly, elsewhere an anti-angiogenic and antimelanoma effect has been reported to be related to a proinflammatory state mediated by pro-inflammatory cytokines such as CXCL10/IP-10 [58]. We then conclude that the activation of pro-inflammatory pathways may underlie, at least to a certain extent, the anti-angiogenic and antimelanoma effect of PDGFR-alpha via CXCL10/IP-10.

The protective role of CXCL10/IP-10 has been already investigated in melanoma [23, 27, 59-61]. The present study for the first time unreveals direct connection with the PDGF-R alpha signalling. In facts, the neutralizing anti-CXCL10/IP-10 antibody was found to revert the anti-proliferation effect in cells overexpressing PDGFR-alpha (Figure 6) further confirming the effects shown in Figures 2 and 3. An additional support was found in vivo in 353 human biopsies samples from http://ist.medisapiens.com/ (Figure 1D). Such data indicate that PDGFR-alpha and CXCL10/IP-10 expression levels show a significant negative correlation $(R=-0.2)$ in healthy skin while it turns to a significant positive correlation $(R=+0.2)$ in melanoma human specimens ( $p<0.001$ in either cases). Finally, when the correlation index was computed on melanoma data reported in Figure 1E, PDGFR-alpha expression levels show a significant positive correlation with CXCL10/IP10 levels $(\mathrm{p}=0.002)$ while PDGFRbeta levels show no significant correlation $(p=0.6)$, further supporting a specific functional link of PDGFRalpha with CXCL10/IP10.

We therefore hypothesized that the biological effects of PDGFR-alpha may be mediated, at least in part, by CXCL10/ IP-10. This cytokine is known to be directly involved in two molecular pathways, namely Cytokine-Cytokine receptor interaction, and Toll-like receptor signaling pathway, according to the CXCL10 Cancer GeneticsWeb card (see http://www.cancerindex.org/geneweb/CXCL10.htm). Both these pathways were predicted to be significantly affected in cells over-expressing PDGFR-alpha, as compared to control cells. This was observed in HUVEC over-expressing PDGFR-alpha (see Table 2) and the first pathway was found to be affected also in SKMel-28 overexpressing PDGFRalpha (see Table 2), confirming that PDGFR-alpha-relatedmay overlap CXCL10/IP-10- related-pathways, including the inflammation-related ones.

Overexpressing PDGFR-alpha has been found in the present study to significantly reduce miR-503 expression. Since we also demonstrate that CXCL10/IP-10 is a validated miR-503 target, down-regulation of this miRNA may explain, at least in part, the observed CXCL10/IP-10 up-regulation. In the recent few years miR-503 has been found to exert opposite effects in different cancer settings; namely it shows a protective role by inhibiting proliferation and inducing apoptosis in glioma [62] and in prostate cancer [63], while on the contrary its down-regulation has shown a protective role in esophagus carcinoma [64] and osteosarcoma [65]. Additional data indicate contrasting effect of miR-503 in colon carcinoma $[66,67]$.

We also found a significant down-regulation of miR424 both in HUVEC and melanoma cells overexpressing PDGF-R alpha. miR-424 has been implicated in angiogenesis regulation $[68,69]$, and suppression of the miR-424-503 gene has been shown to promote mucosal defence in bacterial infection via CX3CL1 expression [70].

Further, we observed that PDGFR-alpha overexpression strongly induces miR-630 expression. This miRNA is known to promote apoptosis and autophagy in cancer cells [71-74]; itis a key negative regulator incancer progression [75] and may represent a further mechanism underlying the anti-proliferation activity of PDGFR-alpha.

In conclusion, the cartoon reported in Figure 9 summarizes the interventions and the cellular reactions observed in the present study. As depicted, over-expressing PDGFR-alpha reduces melanoma and endothelial cell number by affecting several molecular functions (such as Cell-growth and proliferation; Cell Death and Survival, Cell Signaling) via up- and down- regulation of several interconnected molecules. Namely, CXCL10/IP-10, miRNA-503, CEBPB and CEBPD, two known oncosuppressor genes $[50,51,53,54,52]$ were found strongly modified in cells over-expressing PDGFR-alpha, along with other molecules such as CXCL8, IL6, SOCS3, all known to have functional connections with CXCL10/IP-10.

The current study is the first indicating that the anti-melanoma role of PDGFR-alpha may be linked to the known anti-melanoma role of CXCL10/IP-10, and suggests PDGFR-alpha and CXCL10/IP-10 as related targets in melanoma therapy, likely via miR-503.

\section{MATERIALS AND METHODS}

\section{Cell culture}

Human umbilical vein endothelial cells (HUVEC; Clonetics, Lonza) were maintained in complete medium containing endothelial cell basal medium (EBM-2; 
Clonetics, Lonza) supplemented with endothelial cell Bullet Kit (2\% FCS, hEGF-2, hFGF-2, hVEGF, R3IGF-1, ascorbic acid, hydrocortisone, heparin, gentamicin, amphotericin-B; Clonetics, Lonza). Cells were used for the experiments between passage 3rd and 5th. SKMel-28 (ATCC), A375 (ATCC) and Preyer cells (kindly provided by Dr. Tobias Haas, ISS-Rome), were maintained in complete medium Dulbecco's modified Eagle's medium (DMEM; Hyclone, South Logan, UT) supplemented with 10\% FBS (HyClone), $2 \mathrm{mM}$ L-glutamine and $100 \mathrm{IU} / \mathrm{ml}$ penicillin/ streptomycin (Invitrogen, Carlsbad, CA) in humidified 5\% $\mathrm{CO}_{2}$ atmosphere, at $37{ }^{\circ} \mathrm{C}$ as described [76]. The culture medium was changed every 3 days and when cells were subconfluent;monolayers were harvested by 1 minute exposure to $0.1 \%$ Trypsin-EDTA (Life Technologies Inc).

\section{Construction of PDGFR-alpha adenovirus vector}

The recombinant adenoviral vector expressing EGFP and human PDGFR-alpha (AdCMV.PDGFRalpha) and the null (AdCMV.null) control were generated by homologous recombination in bacteria according to a standard procedure [77] as previously reported [31].

\section{Proliferation assay in endothelial- and melanoma cells over-expressing PDGFR-alpha}

HUVEC and SKMel-28 cells $\left(5 \times 10^{5}\right.$ cells in $100 \mathrm{~mm}$ dish) were infected for 2 hours with AdCMV.PDGFR-alpha or with AdCMV.null (10, 30, 100 Multiplicity Of Infection - MOI) in serum-free medium. Cells were then recovered in complete fresh medium and cultured for additional 48 hours. Then cells were harvested and counted with a hemacytometer and total RNA or protein extracts were prepared as reported below as described [78]. The infection-efficiency reached at least $65 \%$ in all cases. For neutralizing antibody experiments: HUVEC and SKMel-28 cells were infected with $30 \mathrm{MOI}$ of AdCMV.PDGFR-alpha or with AdCMV.null for 2 hours, recovered in complete fresh medium and treated with the anti-human CXCL10/IP-10 neutralizing antibody ( $3 \mu \mathrm{g} / \mathrm{ml}$; R\&D Systems, Minneapolis, MN) for 48h. Cells were then harvested and counted as described above. Dasatinib was from Sigma-Aldrich.

\section{PDGFR-alpha expression in vivo by in silico analysis}

IST online is a database reporting gene-expression data from thousands patients of several cancer types, available at IST online Medisapiens (http://www. medisapiens.com/). The platform reportscorrelation dotplots graphs referring to the expression of two genes selected by the operator, indicated in the $\mathrm{X}$ and $\mathrm{Y}$ axes. We investigated PDGFR-alpha gene expression vs the beta 2-microglobulin (taken as a housekeeping gene) in 355 human samples (208 skin melanoma and 147 normal skin). Additional in silico analyses were carried out by accessing the GEO database at (http://www.ncbi.nlm. nih.gov/pubmed) on the GDS3966 dataset, by analysing PDGFR-alpha expression in primary versus metastatic melanoma human biopsies in 83 patients ( 31 primary melanoma and 52 metastatic melanoma) (analysis carried out on GDS3966/203131_at / PDGFRA).

\section{RNA extraction}

RNA extraction from infected cells was obtained as follows: after medium removal, cells were treated with TRIzol reagent (Invitrogen Corporation, Carlsbad, California, USA). Total RNA was isolated from the samples as described earlier [79]. Before use, the RNA concentration in each sample was assayed with NanoDrop 2000C spectrophotometer (NanoDrop, Thermo Scientific, Rockford, IL) and its quality was assessed with an Agilent 2100 Bioanalyzer with the Agilent RNA 6000 nano kit (Agilent Technologies, Santa Clara, CA) as previously described $[80,81]$.

\section{Quantitative Real-time PCR (qRT-PCR)}

According to standard procedures, cDNA was synthesized with Superscript III (Invitrogen-Life Technologies Italia, Monza, Italy) and mRNA expression was analyzed using the SYBR-Green qRT-PCR method (5 ng/assay) (Qiagen, Hilden, Germany) according to the manufacturer's instructions. Quantification was achieved with ABI Prism 7000 SDS (Applied Biosystems, Monza, Italy). mRNA expression value was then normalized for beta 2-microglobulin levels.

Table 2 reports the primers used to this aim. MicroRNA (miRNA) levels were analyzed using the Applied Biosystems TaqMan quantitative qRT-PCR method (1 ng/assay) performed according to the manufacturer's instructions and quantified with the ABI Prism 7000 SDS (Applied Biosystems, Monza, Italy). Mature miRNA levels were normalized to miR-16, whose expression is constant in all tested RNA samples. For both mRNAs and miRNAs, relative expression was calculated using the comparative $\mathrm{Ct}$ method (2- $\Delta \Delta \mathrm{Ct})$.

\section{Gene expression microarray and analysis}

Three independent biological replicates for each condition (HUVEC cells overexpressing or notoverexpressing PDGFR-alpha, and SKMel-28 cells overexpressing or not-overexpressing PDGFR-alpha) were pooled to obtain 4 samples of $500 \mathrm{ng}$ of total RNA as starting material for the synthesis of cDNA and biotinylated cRNA, according to the Illumina TotalPrep RNA Amplification Kit protocol (Ambion, Austin, TX, USA). Then, for each sample, $750 \mathrm{ng}$ of cRNA were hybridized in triplicate on Illumina HumanHT-12 v4.0 BeadChips (Illumina Inc., USA) as already described [82] and subsequently scanned with the Illumina iSCAN. Data 
analyses were performed with GenomeStudio software version 2011.1 (Illumina Inc., USA) as reported in the Statistics section of the Methods. Raw microarray data have been deposited, in a format complying with the Minimum Information about Microarray Gene Experiment guidelines of the Microarray Gene Expression Data Society, in the EBI Array-Express database (www.ebi.ac.uk/arrayexpress) with accession number E-MTAB-4261.

\section{Cytokine quantification}

Fifty microliters of HUVEC and SKMel-28 lysates $(0.8 \mu \mathrm{g}$ total protein $/ \mu \mathrm{l})$ were analyzed to measure cytokine expression with the Bio-Plex Cytokines assay kit (BioRad Laboratories, Milan, Italy), a magnetic-bead based immunoassay. Sample dilution (1:4 in the sampledilution buffer) and handling were carried out strictly according to manufacturer's instructions. After incubation with antibodies-activated magnetic beads, samples were washed using a Bio-Plex ProTM Station (Bio-Rad). The quantification was carried out on a Bio-Plex $® 200$ System (Bio-Rad) run by a Bio-Plex Manager Software version 6.1 and results were expressed as $\mathrm{pg} / \mathrm{ml}$. Normalization of samples was achieved by correcting for total protein concentration of cell lysates. Data are expressed as mean \pm standard deviation (SD). Three independent experiments were carried out.

\section{miRNA microarray and data analysis}

For miRNA expression profiling, three independent biological replicates for each condition (i.e., HUVEC and SKMel-28 cells overexpressing or not PDGFR-alpha) were used and pooled to obtain 4 samples. For each sample, total RNA (800 ng) was used as input for labeling reaction and hybridization according to the miRNA Microarray System with miRNA Complete Labeling and Hyb Kit protocol (Agilent Technologies). Labeled RNAs were then hybridized in technical triplicate on Agilent human miRNA microarray rel. 14, V2, 8x15K (Agilent Technologies) and acquired by using Agilent Microarray Scanner G2565BA (Agilent Technologies). Data analysis was performed with Agilent Feature Extraction v.10.7.3.1, Gene spring v.11.5 (Agilent Technologies). Data were analyzed as reported in Statistics section of Methods. Raw miRNA microarray data for HUVEC and SKMel-28 cells have been deposited, in a format complying with the Minimum Information about Microarray Gene Experiment guidelines of the Microarray Gene Expression Data Society, in the EBI Array-Express database (www.ebi.ac.uk/arrayexpress) with accession number E-MTAB-4266.

\section{Luciferase reporter assay}

CXCL10/IP-10 3'UTR Luciferase Stable 293 Cell Line (purchased from abm) containing target site sequences complementary to the seed sequence of miR-503 cloned downstream of the luciferase gene was used. Moreover, 27 nucleotides deleted sequence (5'-tgatggtcaccaaatcagctgctacta $\left.-3^{\prime}\right)$, at 541 to 567 3'UTR of human CXCL10/IP-10 Luciferase Stable 293 Cell Line and Blank (Control) 3'UTR Luciferase Stable 293 Cell Line were also used (abm). For the reporter assay, cells were transiently transfected with $800 \mathrm{ng}$ pEP-miR-503 or with pEP-miR-Null Control (Cell Biolabs, inc.) using FuGENE 6 Transfection Reagent (Roche). Each sample was co-transfected with $80 \mathrm{ng}$ of pRL-TK plasmid expressing Renilla luciferase (Promega, Madison). Cell collection was carried out $48 \mathrm{~h}$ after transfection and analyzed using the Dual-Luciferase Reporter Assay System (Promega, Madison). Relative luciferase activity was normalized to Renilla luciferase activity. Transfections were performed in triplicate and repeated at least 3 times in independent experiments.

\section{Bioinformatic analysis of genes and miRNAs expression profiling}

The list of genes differentially expressed has been analyzed for Gene Ontology terms enrichment and KEGG pathway involvement by means of DAVID v. 6.7 online tool (Database for Annotation, Visualization and Integrated Discovery, at https://david.ncifcrf.gov/). Lists of up- and down-regulated genes were analyzed with reference to background list consisting of the detected genes in the same experimental conditions in control cells. For miR503, putative mRNA targets prediction was carried out using TargetScan software [83-86].

TargetScan provides computationally predicted miRNA gene targets by searching for the presence of 8-mer and 7-mer sites matching the seed region of each miRNA.

The list of modified transcripts was also analyzed using Ingenuity Pathway Analysis Software (IPA, Ingenuity ${ }^{\circledR}$ Systems, www.ingenuity.com). It refers to a proprietary knowledge base (Ingenuity Pathways Knowledge Base) where biological interactions and functional properties are annotated. IPA Functional Analysis on "molecular and cellular functions" category and Canonical Pathway investigation were carried out calculating the likelihood that the association between a given transcription dataset and a specific function or pathway is due to random choice, and it is expressed as a $p$ value calculated using the right-tailed Fisher Exact Test. For network generation, each differentially expressed transcript identifier was uploaded and mapped to its corresponding object in Ingenuity Knowledge Base to algorithmically generate molecular networks based on their connectivity. Obtained networks are scored according to a numerical value (Score) taking into account the number of dataset molecules they contain and the network size as well as the total number of input transcripts in the dataset and the total number of molecules 
in the Ingenuity Knowledge Base that could potentially be included in the networks. The network Score is based on the hypergeometric distribution and is calculated with the right-tailed Fisher Exact Test. Finally, the "microRNA Target Filter" tool was used to provide insights into the biological effects of microRNAs, using experimentally validated interactions from TarBase and miRecords, as well as predicted microRNA-mRNA interactions from TargetScan examining microRNA-mRNA pairings in the pathways of interest.

\section{Statistical analysis}

Proliferation data, qRT-PCR data, cytokine expression and luciferase activity data were analyzed with GraphPad Prism 4.0 software packages (Graph Pad Inc. San Diego, CA). A statistical significance threshold of $p<0.05$ was considered. Normal distribution of datasets was tested according to D'Agostino and Pearson omnibus normality test. A 2 tails unpaired t Test assay was then performed if normality test was passed. A non-parametric Mann-Whitney test was carried out if D'Agostino and Pearson normality test was not passed. Benjamini test was carried to identify pathways significantly affected in over-expressing cells. For gene expression analysis, data were normalized with the quantile algorithm, and genes were considered detected if the detection $p$-value was less than 0.05. Statistical significance was calculated with Illumina DiffScore, a proprietary algorithm that uses the bead standard deviation to build an error model. Only genes with at least 1.5 fold increase or decrease and DiffScore of $\leq-30$ or $\geq 30$, corresponding to a $p$-value cut off $\leq 0.001$, were considered as statistically significant by comparing all values obtained in HUVEC cells overexpressing PDGFR-alpha vs HUVEC control cells as well as SKMel-28 cells overexpressing PDGFR-alpha vs SKMel-28 control cells.

For miRNA microarray data analysis, a quantilenormalization was applied and statistical significance was calculated by using the unpaired t-Test for two groups. Only miRNAs with at least 1.5 fold increase or decrease and $p$ value cut-off $\leq 0.05$, were considered as differentially expressed with statistical significance, by comparing all values obtained in HUVEC cells overexpressing PDGFRalpha vs HUVEC control cells as well as SKMel-28 cells overexpressing PDGFR-alpha vs SKMel-28 control cells.

\section{ACKNOWLEDGMENTS}

Authors are grateful to the Proteomic Facility for Complex Protein Mixture (CPM) Analysis, the Telethon Proteomics Service (Project number: GTF08002) at Istituto Superiore di Sanità, Rome, and Genomix4Life Srl.

\section{CONFLICTS OF INTEREST}

No potential conflicts of interest were disclosed.

\section{GRANT SUPPORT}

This work was supported by the Italian Ministry of Health (grant RC12 3.5, RC2015 3.4 and Young Researcher Grant GR-2011-02347781and RF07ONC-25/3), the ItalyUSA Oncoproteomics Program of the Italian Ministry of Health, the Italian Association for Cancer Research (AIRC grant IG-17426) and the National Research Council of Italy (CNR Flagship Project InterOmics).

Tabolacci C. was recipient of two fellowships funded by Fondazione Umberto Veronesi, gratefully acknowledged.

\section{Authors' contributions}

Conception and design: D. D'Arcangelo, F. Facchiano, A. Facchiano

Development of methodology: D. D'Arcangelo, S. Rossi, A. Salvati, G. Nassa, A. Weisz

Acquisition of data (proliferation assays, cells infection; adenovirus production, ELISA analyses, reagents handling, qRT-PCR assays): D. D'Arcangelo, S. Rossi, A. Antonini, A. Stancato, A. Salvati, G. Nassa, M. Cordella

Analysis and interpretation of data (e.g., statistical analysis, biostatistics, computational analysis): D. D’Arcangelo, F. Facchiano, A. Facchiano, A.M. Facchiano, G. Nassa, A. Salvati, R. Tarallo, A. Weisz

Writing, review, and/or revision of the manuscript: D. D'Arcangelo, F. Facchiano, A. Facchiano, AM. Facchiano, A. Weisz, G. Nassa, R. Tarallo

Administrative, technical, or material support (i.e., reporting or organizing data, constructing databases): S. Rossi, G Nassa, C. Senatore, C. Tabolacci, A. Antonini, A. Stancato Weisz

Study supervision: F. Facchiano, A. Facchiano, A.

\section{REFERENCES}

1. Palmieri G, Capone M, Ascierto ML, Gentilcore G, Stroncek DF, Casula M, Sini MC, Palla M, Mozzillo N, Ascierto PA. Main roads to melanoma. J Transl Med 2009; 7:86.

2. Bränström R, Chang Y-M, Kasparian N, Affleck P, Tibben A, Aspinwall LG, Azizi E, Baron-Epel O, Battistuzzi L, Bruno W, Chan M, Cuellar F, et al. Melanoma risk factors, perceived threat and intentional tanning: an international online survey. Eur J Cancer Prev 2010; 19:216-26.

3. Burkhart CG, Burkhart CN. Melanoma risk factors include insecticides and occupational exposures. Int J Dermatol 2009; 48:210.

4. Postow MA, Harding J, Wolchok JD. Targeting immune checkpoints: releasing the restraints on anti-tumor immunity for patients with melanoma. Cancer J 2013; 18:153-9.

5. Funt SA, Page DB, Wolchok JD, Postow MA. CTLA-4 antibodies: new directions, new combinations. Oncology (Williston Park) 2014; 28 Suppl 3:6-14. 
6. Christiaansen A, Varga SM, Spencer J V. Viral manipulation of the host immune response. Curr Opin Immunol 2015; 36:54-60.

7. Scolletta S, Colletti M, Di Luigi L, Crescioli C. Vitamin $\mathrm{D}$ receptor agonists target CXCL10: new therapeutic tools for resolution of inflammation. Mediators Inflamm 2013; 2013:876319.

8. Mazzi V, Ferrari SM, Giuggioli D, Antonelli A, Ferri C, Fallahi P. Role of CXCL10 in cryoglobulinemia. Clin Exp Rheumatol 2015; 33:433-6.

9. Melnikova VO, Bar-Eli M. Inflammation and melanoma metastasis. Pigment Cell Melanoma Res 2009; 22:257-67.

10. Dunn JH, Ellis LZ, Fujita M. Inflammasomes as molecular mediators of inflammation and cancer: Potential role in melanoma. Cancer Lett 2012; 314:24-33.

11. Sanz-Motilva V, Martorell-Calatayud A, Nagore E. Nonsteroidal anti-inflammatory drugs and melanoma. Curr Pharm Des 2012; 18:3966-78.

12. Navarini-Meury AA, Conrad C. Melanoma and innate immunity--aActive inflammation or just erroneous attraction? Melanoma as the source of leukocyte-attracting chemokines. Semin Cancer Biol 2009; 19:84-91.

13. Hsiao JJ, Fisher DE. The roles of microphthalmia-associated transcription factor and pigmentation in melanoma. Arch Biochem Biophys 2014; 563:28-34.

14. Kaufman HL, Ruby CE, Hughes T, Slingluff CL. Current status of granulocyte-macrophage colony-stimulating factor in the immunotherapy of melanoma. J Immunother Cancer 2014; 2:11.

15. Mann J, Thomson P, Stevens H, Palamaras I. Malignant melanoma and tumor necrosis factor- $\alpha$ inhibitors: a case report and review of the literature. Int J Dermatol 2013; 52:471-4.

16. Lasfar A, Cohen-Solal KA. Resistance to transforming growth factor $\beta$-mediated tumor suppression in melanoma: are multiple mechanisms in place? Carcinogenesis 2010; 31:1710-7.

17. Lindell DM, Lane TE, Lukacs NW. CXCL10/CXCR3mediated responses promote immunity to respiratory syncytial virus infection by augmenting dendritic cell and CD8(+) T cell efficacy. Eur J Immunol 2008; 38:2168-79.

18. Fujita T, Matsuoka T, Honda T, Kabashima K, Hirata T, Narumiya S. A GPR40 agonist GW9508 suppresses CCL5, CCL17, and CXCL10 induction in keratinocytes and attenuates cutaneous immune inflammation. J Invest Dermatol 2011; 131:1660-7.

19. Oliver BG, Price P, Wand H, French MA. Increased plasma CXCL10 may be a marker of increased risk of immune restoration disease associated with nonviral pathogens. J Acquir Immune Defic Syndr 2012; 59:e47-9.

20. Bondar C, Araya RE, Guzman L, Rua EC, Chopita N, Chirdo FG. Role of CXCR3/CXCL10 axis in immune cell recruitment into the small intestine in celiac disease. PLoS One 2014; 9:e89068.
21. Brownell J, Polyak SJ. Molecular pathways: hepatitis C virus, CXCL10, and the inflammatory road to liver cancer. Clin Cancer Res 2013; 19:1347-52.

22. Polimeno M, Napolitano M, Costantini S, Portella L, Esposito A, Capone F, Guerriero E, Trotta A, Zanotta S, Pucci L, Longo N, Perdonà S, et al. Regulatory T cells, interleukin (IL)-6, IL-8, vascular endothelial growth factor (VEGF), CXCL10, CXCL11, epidermal growth factor (EGF) and hepatocyte growth factor (HGF) as surrogate markers of host immunity in patients with renal cell carcinoma. BJU Int 2013; 112:686-96.

23. Antonicelli F, Lorin J, Kurdykowski S, Gangloff SC, Le Naour R, Sallenave JM, Hornebeck W, Grange F, Bernard P. CXCL10 reduces melanoma proliferation and invasiveness in vitro and in vivo. Br J Dermatol 2011; 164:720-8.

24. Mohty A-M, Grob J-J, Mohty M, Richard M-A, Olive D, Gaugler B. Induction of IP-10/CXCL10 secretion as an immunomodulatory effect of low-dose adjuvant interferonalpha during treatment of melanoma. Immunobiology 2010; 215:113-23.

25. Feldman ED, Weinreich DM, Carroll NM, Burness ML, Feldman AL, Turner E, Xu H, Alexander HR. Interferon gamma-inducible protein 10 selectively inhibits proliferation and induces apoptosis in endothelial cells. Ann Surg Oncol 2006; 13:125-33.

26. Sgadari C, Angiolillo AL, Tosato G. Inhibition of angiogenesis by interleukin-12 is mediated by the interferon-inducible protein 10. Blood 1996; 87:3877-82.

27. Tanese K, Grimm EA, Ekmekcioglu S. The role of melanoma tumor-derived nitric oxide in the tumor inflammatory microenvironment: its impact on the chemokine expression profile, including suppression of CXCL10. Int J Cancer 2012; 131:891-901.

28. Facchiano A, De Marchis F, Turchetti E, Facchiano F, Guglielmi M, Denaro A, Palumbo R, Scoccianti M, Capogrossi MC. The chemotactic and mitogenic effects of platelet-derived growth factor-BB on rat aorta smooth muscle cells are inhibited by basic fibroblast growth factor. J Cell Sci 2000; 113 ( Pt 16):2855-2863.

29. De Marchis F. Platelet-derived growth factor inhibits basic fibroblast growth factor angiogenic properties in vitro and in vivo through its alpha receptor. Blood 2002; 99:2045-2053.

30. Faraone D, Aguzzi MS, Ragone G, Russo K, Capogrossi $\mathrm{MC}$, Facchiano A. Heterodimerization of FGF-receptor 1 and PDGF-receptor- alpha: A novel mechanism underlying the inhibitory effect of PDGF-BB on FGF-2 in human cells. Blood 2006; 107:1896-1902.

31. Faraone D, Aguzzi MS, Toietta G, Facchiano AM, Facchiano F, Magenta A, Martelli F, Truffa S, Cesareo E, Ribatti D, Capogrossi MC, Facchiano A. Platelet-Derived Growth Factor-Receptor $\alpha$ Strongly Inhibits Melanoma Growth In Vitro and In Vivo. Neoplasia 2009; 11:732 - 42.

32. Aguzzi MS, Faraone D, D'Arcangelo D, De Marchis F, Toietta G, Ribatti D, Parazzoli A, Colombo P, Capogrossi 
MC, Facchiano A. The FGF-2-derived peptide FREG inhibits melanoma growth in vitro and in vivo. Mol Ther 2011; 19:266-73.

33. Yu J, Ustach C, Kim H-RC. Platelet-derived growth factor signaling and human cancer. J Biochem Mol Biol 2003; 36:49-59.

34. Heldin CH, Ostman A, Rönnstrand L. Signal transduction via platelet-derived growth factor receptors. Biochim Biophys Acta 1998; 1378:F79-113.

35. Najy AJ, Won JJ, Movilla LS, Kim H-RC. Differential tumorigenic potential and matriptase activation between PDGF B versus PDGF D in prostate cancer. Mol Cancer Res 2012; 10:1087-97.

36. Rebecca VW, Wood E, Fedorenko I V, Paraiso KHT, Haarberg HE, Chen Y, Xiang Y, Sarnaik A, Gibney GT, Sondak VK, Koomen JM, Smalley KSM. Evaluating melanoma drug response and therapeutic escape with quantitative proteomics. Mol Cell Proteomics 2014; 13:1844-54.

37. Zhang C, Yang C, Wang R, Jiao Y, Ampah KK, Wang X, Zeng X. c-Abl Kinase Is a Regulator of $\alpha v \beta 3$ Integrin Mediated Melanoma A375 Cell Migration. PLoS One 2013; 8:e66108.

38. Wäster P, Rosdahl I, Gilmore BF, Seifert O. Ultraviolet exposure of melanoma cells induces fibroblast activation protein- $\alpha$ in fibroblasts: Implications for melanoma invasion. Int J Oncol 2011; 39:193-202.

39. Ogawa Y, Kawamura T, Furuhashi M, Tsukamoto K, Shimada S. Improving chemotherapeutic drug penetration in melanoma by imatinib mesylate. J Dermatol Sci 2008; 51:190-9.

40. Suzuki S, Heldin C-H, Heuchel RL. Platelet-derived growth factor receptor-beta, carrying the activating mutation D849N, accelerates the establishment of B16 melanoma. BMC Cancer 2007; 7:224.

41. Montero JC, Seoane S, Ocaña A, Pandiella A. Inhibition of SRC family kinases and receptor tyrosine kinases by dasatinib: possible combinations in solid tumors. Clin Cancer Res 2011; 17:5546-52.

42. Knight LA, Di Nicolantonio F, Whitehouse PA, Mercer SJ, Sharma S, Glaysher S, Hungerford JL, Hurren J, Lamont A, Cree IA. The effect of imatinib mesylate (Glivec) on human tumor-derived cells. Anticancer Drugs 2006; 17:649-55.

43. Labala S, Mandapalli PK, Kurumaddali A, Venuganti VVK. Layer-by-layer polymer coated gold nanoparticles for topical delivery of imatinib mesylate to treat melanoma. Mol Pharm 2015; 12:878-88.

44. Willenberg A, Saalbach A, Simon JC, Anderegg U. Melanoma cells control HA synthesis in peritumoral fibroblasts via PDGF-AA and PDGF-CC: impact on melanoma cell proliferation. J Invest Dermatol 2012; 132:385-93.

45. Pirraco A, Coelho P, Rocha A, Costa R, Vasques L, Soares R. Imatinib targets PDGF signaling in melanoma and host smooth muscle neighboring cells. J Cell Biochem 2010; 111:433-41.
46. Corless CL, Schroeder A, Griffith D, Town A, McGreevey L, Harrell P, Shiraga S, Bainbridge T, Morich J, Heinrich MC. PDGFRA mutations in gastrointestinal stromal tumors: frequency, spectrum and in vitro sensitivity to imatinib. J Clin Oncol 2005; 23:5357-64.

47. Luo J-C, Peng Y-L, Chen T-S, Huo T-I, Hou M-C, Huang $\mathrm{H}-\mathrm{C}$, Lin $\mathrm{H}-\mathrm{C}$, Lee F-Y. Clopidogrel inhibits angiogenesis of gastric ulcer healing via downregulation of vascular endothelial growth factor receptor 2. J Formos Med Assoc 2016; 115:764-72.

48. Russo K, Ragone R, Facchiano AM, Capogrossi MC, Facchiano A. Platelet-derived growth factor-BB and basic fibroblast growth factor directly interact in vitro with high affinity. J Biol Chem 2002; 277:1284-91.

49. Xu L, Shen SS, Hoshida Y, Subramanian A, Ross K, Brunet J-P, Wagner SN, Ramaswamy S, Mesirov JP, Hynes RO. Gene expression changes in an animal melanoma model correlate with aggressiveness of human melanoma metastases. Mol Cancer Res 2008; 6:760-9.

50. Brownell J, Bruckner J, Wagoner J, Thomas E, Loo Y-M, Gale M, Liang TJ, Polyak SJ. Direct, interferonindependent activation of the CXCL10 promoter by NF- $\mathrm{KB}$ and interferon regulatory factor 3 during hepatitis $\mathrm{C}$ virus infection. J Virol 2014; 88:1582-90.

51. Chuang C-H, Wang W-J, Li C-F, Ko C-Y, Chou Y-H, Chuu C-P, Cheng T-L, Wang J-M. The combination of the prodrugs perforin-CEBPD and perforin-granzyme $\mathrm{B}$ efficiently enhances the activation of caspase signaling and kills prostate cancer. Cell Death Dis 2014; 5:e1220.

52. Fang T, Cui M, Sun J, Ge C, Zhao F, Zhang L, Tian H, Zhang L, Chen T, Jiang G, Xie H, Cui Y, et al. Orosomucoid 2 inhibits tumor metastasis and is upregulated by CCAAT/enhancer binding protein $\beta$ in hepatocellular carcinomas. Oncotarget 2015; 6:16106-19. doi: 10.18632/oncotarget.3867.

53. Li C-F, Tsai H-H, Ko C-Y, Pan Y-C, Yen C-J, Lai H-Y, Yuh $\mathrm{C}-\mathrm{H}, \mathrm{Wu}$ W-C, Wang J-M. HMDB and 5-AzadC Combination Reverses Tumor Suppressor CCAAT/ Enhancer-Binding Protein Delta to Strengthen the Death of Liver Cancer Cells. Mol Cancer Ther 2015; 14:2623-33.

54. Li M, Zhou W, Yuan R, Chen L, Liu T, Huang D, Hao L, Xie Y, Shao J. ROCK2 promotes HCC proliferation by CEBPD inhibition through phospho-GSK3 $\beta / \beta$-catenin signaling. FEBS Lett 2015; 589:1018-25.

55. Facchiano A, Russo K, Facchiano AM, De Marchis F, Facchiano F, Ribatti D, Aguzzi MS, Capogrossi MC. Identification of a novel domain of fibroblast growth factor 2 controlling its angiogenic properties. J Biol Chem 2003; 278:8751-8760.

56. Aguzzi MS, D'Arcangelo D, Giampietri C, Capogrossi MC, Facchiano A. RAM, an RGDS analog, exerts potent anti-melanoma effects in vitro and in vivo. PLoS One 2011; 6:e25352.

57. Salem ML, Attia ZI, Galal SM. Acute inflammation induces immunomodulatory effects on myeloid cells associated with 
anti-tumor responses in a tumor mouse model. J Adv Res 2016; 7:243-53.

58. Huang H, Langenkamp E, Georganaki M, Loskog A, Fuchs PF redlund, Dieterich LC, Kreuger J, Dimberg A. VEGF suppresses T-lymphocyte infiltration in the tumor microenvironment through inhibition of NFkB-induced endothelial activation. FASEB J 2015; 29:227-238.

59. Kobayashi H, Nobeyama Y, Nakagawa H. Tumorsuppressive effects of natural-type interferon- $\beta$ through CXCL10 in melanoma. Biochem Biophys Res Commun 2015; 464:416-21.

60. Mauldin IS, Wang E, Deacon DH, Olson WC, Bao Y, Slingluff CL. TLR2/6 agonists and interferon-gamma induce human melanoma cells to produce CXCL10. Int J Cancer 2015; 137:1386-96.

61. Smoller BR, Krueger J. Detection of cytokine-induced protein gamma-immune protein-10 (gamma-IP10) in atypical melanocytic proliferations. J Am Acad Dermatol 1991; 25:627-31

62. Liu H, Song Z, Liao D, Zhang T, Liu F, Zheng W, Luo K, Yang L. miR-503 inhibits cell proliferation and invasion in glioma by targeting L1CAM. Int J Clin Exp Med 2015; 8:18441-7.

63. Guo J, Liu X, Wang M. miR-503 suppresses tumor cell proliferation and metastasis by directly targeting RNF31 in prostate cancer. Biochem Biophys Res Commun 2015; 464:1302-8.

64. Zhao K, Chen B-J, Chen Z-G, Zhang Y-J, Xu D, Liu Q. Effect of miR-503 Down-Regulation on Growth and Invasion of Esophagus Carcinoma and Related Immune Function. Med Sci Monit 2015; 21:3564-9.

65. Bassampour SA, Abdi R, Bahador R, Shakeri M, Torkaman A, Yahaghi E, Taheriazam A. Downregulation of miR133b/miR-503 acts as efficient prognostic and diagnostic factors in patients with osteosarcoma and these predictor biomarkers are correlated with overall survival. Tumour Biol 2015.

66. Chang S-W, Yue J, Wang B-C, Zhang X-L. miR-503 inhibits cell proliferation and induces apoptosis in colorectal cancer cells by targeting E2F3. Int J Clin Exp Pathol 2015; $8: 12853-60$.

67. Li L, Sarver AL, Khatri R, Hajeri PB, Kamenev I, French AJ, Thibodeau SN, Steer CJ, Subramanian S. Sequential expression of miR-182 and miR-503 cooperatively targets FBXW7, contributing to the malignant transformation of colon adenoma to adenocarcinoma. J Pathol 2014; 234:488-501.

68. Ghosh G, Subramanian I V, Adhikari N, Zhang X, Joshi HP, Basi D, Chandrashekhar YS, Hall JL, Roy S, Zeng Y, Ramakrishnan S. Hypoxia-induced microRNA-424 expression in human endothelial cells regulates HIF- $\alpha$ isoforms and promotes angiogenesis. J Clin Invest 2010; 120:4141-54.

69. Chamorro-Jorganes A, Araldi E, Penalva LOF, Sandhu D, Fernández-Hernando C, Suárez Y. MicroRNA-16 and microRNA-424 regulate cell-autonomous angiogenic functions in endothelial cells via targeting vascular endothelial growth factor receptor-2 and fibroblast growth factor receptor-1. Arterioscler Thromb Vasc Biol 2011; 31:2595-606.

70. Zhou R, Gong A-Y, Chen D, Miller RE, Eischeid AN, Chen $\mathrm{X}-\mathrm{M}$. Histone deacetylases and NF-kB signaling coordinate expression of CX3CL1 in epithelial cells in response to microbial challenge by suppressing miR-424 and miR-503. PLoS One 2013; 8:e65153.

71. Farhana L, Dawson MI, Murshed F, Das JK, Rishi AK, Fontana JA. Upregulation of miR-150* and miR-630 induces apoptosis in pancreatic cancer cells by targeting IGF-1R. PLoS One 2013; 8:e61015.

72. Cao J-X, Lu Y, Qi J-J, An G-S, Mao Z-B, Jia H-T, Li S-Y, Ni J-H. MiR-630 inhibits proliferation by targeting CDC7 kinase, but maintains the apoptotic balance by targeting multiple modulators in human lung cancer A549 cells. Cell Death Dis 2014; 5:e1426.

73. Huang Y, Guerrero-Preston R, Ratovitski EA. Phospho$\Delta \mathrm{Np} 63 \alpha$-dependent regulation of autophagic signaling through transcription and micro-RNA modulation. Cell Cycle 2012; 11:1247-59.

74. Jing Z, Han W, Sui X, Xie J, Pan H. Interaction of autophagy with microRNAs and their potential therapeutic implications in human cancers. Cancer Lett 2015; 356:332-8.

75. Corcoran C, Rani S, Breslin S, Gogarty M, Ghobrial IM, Crown J, O'Driscoll L. miR-630 targets IGF1R to regulate response to HER-targeting drugs and overall cancer cell progression in HER2 over-expressing breast cancer. Mol Cancer 2014; 13:71.

76. Facchiano F, D'Arcangelo D, Lentini A, Rossi S, Senatore C, Pannellini T, Tabolacci C, Facchiano AM, Facchiano A, Beninati S. Tissue transglutaminase activity protects from cutaneous melanoma metastatic dissemination: An in vivo study. Amino Acids 2013; 44:53-61.

77. He TC, Zhou S, da Costa LT, Yu J, Kinzler KW, Vogelstein B. A simplified system for generating recombinant adenoviruses. Proc Natl Acad Sci U S A 1998; 95:2509-14.

78. D'Arcangelo D, Gaetano C, Capogrossi MC. Acidification Prevents Endothelial Cell Apoptosis by Axl Activation. Circ Res 2002; 91:4e-12.

79. Ferraro L, Ravo M, Nassa G, Tarallo R, De Filippo MR, Giurato G, Cirillo F, Stellato C, Silvestro S, Cantarella C, Rizzo F, Cimino D, et al. Effects of oestrogen on microRNA expression in hormone-responsive breast cancer cells. Horm Cancer 2012; 3:65-78.

80. Grober OM V, Mutarelli M, Giurato G, Ravo M, Cicatiello L, De Filippo MR, Ferraro L, Nassa G, Papa MF, Paris O, Tarallo R, Luo S, et al. Global analysis of estrogen receptor beta binding to breast cancer cell genome reveals an extensive interplay with estrogen receptor alpha for target gene regulation. BMC Genomics 2011; 12:36.

81. Nassa G, Tarallo R, Giurato G, De Filippo MR, Ravo M, Rizzo F, Stellato C, Ambrosino C, Baumann M, Lietzèn N, Nyman TA, Weisz A. Post-transcriptional regulation of human breast 
cancer cell proteome by unliganded estrogen receptor $\beta$ via microRNAs. Mol Cell Proteomics 2014; 13:1076-90.

82. Cirillo F, Nassa G, Tarallo R, Stellato C, De Filippo MR, Ambrosino C, Baumann M, Nyman TA, Weisz A. Molecular mechanisms of selective estrogen receptor modulator activity in human breast cancer cells: identification of novel nuclear cofactors of antiestrogen-ER $\alpha$ complexes by interaction proteomics. J Proteome Res 2013; 12:421-31.

83. Lewis BP, Burge CB, Bartel DP. Conserved seed pairing, often flanked by adenosines, indicates that thousands of human genes are microRNA targets. Cell 2005; 120:15-20.
84. Grimson A, Farh KK-H, Johnston WK, Garrett-Engele P, Lim LP, Bartel DP. MicroRNA targeting specificity in mammals: determinants beyond seed pairing. Mol Cell 2007; 27:91-105.

85. Friedman RC, Farh KK-H, Burge CB, Bartel DP. Most mammalian mRNAs are conserved targets of microRNAs. Genome Res 2009; 19:92-105.

86. Garcia DM, Baek D, Shin C, Bell GW, Grimson A, Bartel DP. Weak seed-pairing stability and high targetsite abundance decrease the proficiency of 1sy- 6 and other microRNAs. Nat Struct Mol Biol 2011; 18:1139-46. 